\title{
Charge Transfer from Photoexcited Semiconducting Single-Walled Carbon Nanotubes to Wide-Bandgap Wrapping Polymer
}

Zhuoran Kuang, Felix J. Berger, Jose Luis Pérez Lustres, Nikolaus Wollscheid, Han Li, Jan Lüttgens, Merve Balcı Leinen, Benjamin S. Flavel, Jana Zaumseil, and Tiago Buckup*

Cite This: J. Phys. Chem. C 2021, 125, 8125-8136

Read Online

ACCESS

Wll Metrics \& More

Article Recommendations

Supporting Information

ABSTRACT: As narrow optical bandgap materials, semiconducting singlewalled carbon nanotubes (SWCNTs) are rarely regarded as charge donors in photoinduced charge-transfer (PCT) reactions. However, the unique band structure and unusual exciton dynamics of SWCNTs add more possibilities to the classical PCT mechanism. In this work, we demonstrate PCT from photoexcited semiconducting $(6,5)$ SWCNTs to a wide-bandgap wrapping poly-[(9,9-dioctylfluorenyl-2,7-diyl)-alt-(6,6')-(2,2'-bipyridine)] (PFO$\mathrm{BPy})$ via femtosecond transient absorption spectroscopy. By monitoring the spectral dynamics of the SWCNT polaron, we show that charge transfer from photoexcited SWCNTs to PFO-BPy can be driven not only by the energetically favorable $\mathrm{E}_{33}$ transition but also by the energetically unfavorable $\mathrm{E}_{22}$ excitation under high pump fluence. This unusual PCT from narrow-

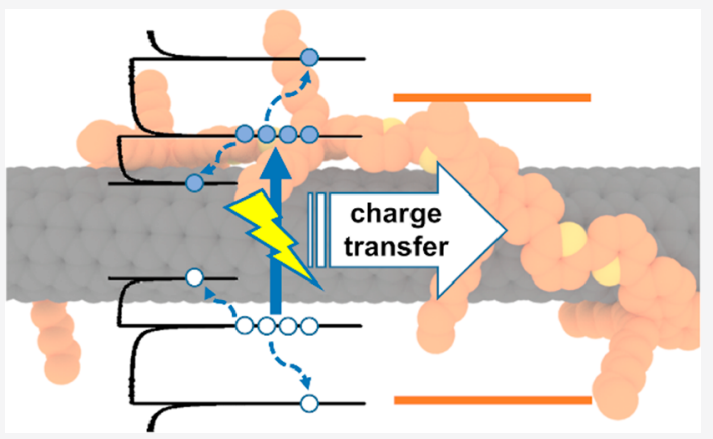
bandgap SWCNTs toward a wide-bandgap polymer originates from the up-converted high-energy excitonic state $\left(\mathrm{E}_{33}\right.$ or higher) that is promoted by the Auger recombination of excitons and charge carriers in SWCNTs. These insights provide new pathways for charge separation in SWCNT-based photodetectors and photovoltaic cells.

\section{INTRODUCTION}

The highly selective wrapping of semiconducting single-walled carbon nanotubes (SWCNTs) with conjugated polymers, e.g., polyfluorenes and polythiophenes, has enabled monochiral samples with high purity ${ }^{1-3}$ to be available for a wide range of optoelectronic applications from field-effect transistors, ${ }^{4}$ lightemitting diodes, ${ }^{5}$ and electrochromic cells ${ }^{6}$ to photovoltaic cells ${ }^{7,8}$ and photodiodes. ${ }^{9}$ The interaction of nanotubes with their immediate environment, i.e., the wrapping polymer or matrix, with regard to energy and charge transfer depending on the energy level alignment ${ }^{10,11}$ is crucial for their functionality and not yet fully understood. The unique electronic structure and complex photophysics of SWCNTs ${ }^{12}$ make this interaction highly interesting from a fundamental and application point of view. For photovoltaic cells based on carbon nanotubes, SWCNTs assume the role of donor in photoinduced charge transfer (PCT) only when a semiconductor with high electron affinity (e.g., fullerene derivatives) acts as an acceptor. ${ }^{13-15}$ When wrapped by typical semiconducting polymers, the narrow-bandgap nanotubes usually behave as acceptors for both charges and excitation. ${ }^{16-21}$

Various spectroscopic methods, including pump-probe transient absorption (TA) and transient fluorescence spectroscopy, have been applied to explore the excited-state interactions between SWCNTs and the conjugated wrapping polymer. ${ }^{11,16,22-25}$ Strong electronic interaction in SWCNT/ polymer hybrids has been concluded by studying the modulation effect of the polymer frontier orbital levels on nanotube valence and conduction band energies, as well as newly formed hybridized electronic states between the two components. $^{22,23,25}$ The energy transfer observed in SWCNT/ polymer composites also points to an electron-exchange mechanism. ${ }^{11,16}$ However, only the first optical bandgap in semiconductors is usually taken into account in studies of the classical PCT mechanism. The electronic interplay between the complex band structure of SWCNTs and the wrapping polymer has not been clarified yet. ${ }^{26,27}$

$(6,5)$ SWCNTs are chosen for this study due to their availability as nearly monochiral samples in large amounts after selective dispersion with a polyfluorene-bipyridine copolymer (PFO-BPy). ${ }^{2}$ The energy level alignment of $(6,5)$ SWCNTs and $\mathrm{PFO}-\mathrm{BPy}$ (Figure 1) indicates that the PFO-BPywrapped $(6,5)$ SWCNT hybrid system (hereafter referred to as Hybrid) constitutes a Type-I heterojunction. When regarding the narrow-bandgap $(6,5)$ SWCNT as a charge donor, the PCT from excited $(6,5)$ SWCNT toward wide-bandgap acceptor PFO-BPy is energetically unfavorable, at least for

Received: November 11, 2020

Revised: March 16, 2021

Published: April 14, 2021 


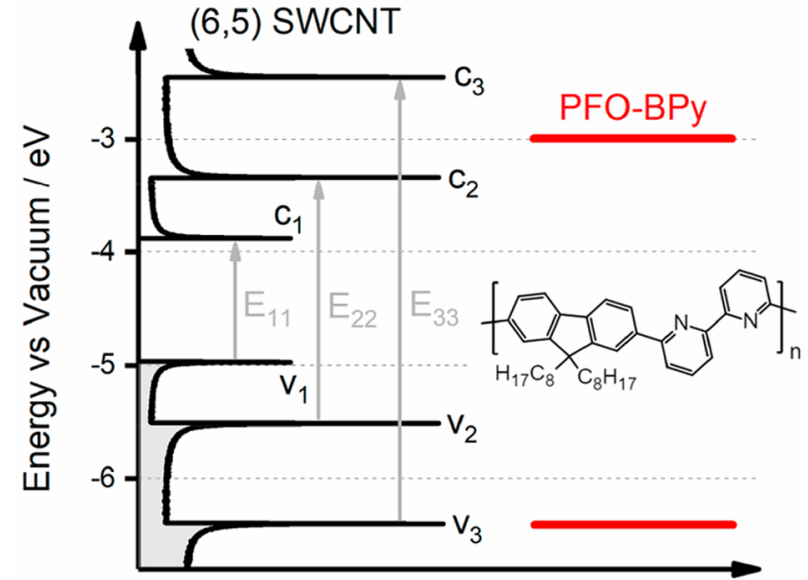

Density of States

Figure 1. Schematic energy level alignment of $(6,5)$ SWCNT and PFO-BPy. The density of states of $(6,5)$ SWCNT with characteristic van Hove singularities of the valence $\left(\mathrm{v}_{1}, \mathrm{v}_{2}, \mathrm{v}_{3}\right)$ and conduction $\left(\mathrm{c}_{1}\right.$, $c_{2}, c_{3}$ ) band was based on ref 28 and shifted by the reported ionization potential. ${ }^{16}$ The HOMO and LUMO energies of PFO-BPy indicated by red horizontal lines were reported by Jang et al. ${ }^{29}$ The gray arrows are simplified representations for observed excitonic absorption bands $\mathrm{E}_{11}, \mathrm{E}_{22}$, and $\mathrm{E}_{33}$. The inset shows the molecular structure of PFOBPy.

the $\mathrm{E}_{11}$ and $\mathrm{E}_{22}$ transitions. However, the established exciton photophysics of SWCNTs suggests that low-energy photon excitation is able to promote populations of high-energy excitonic states via, e.g., Auger recombination of excitons. ${ }^{30-36}$ Under this mechanism, even in the presence of ultrafast intersubband relaxation, high-energy excitonic states still have a considerable decay time as that of the $\mathrm{E}_{11}$ state, which may favor the PCT from excited SWCNTs to a wide-bandgap acceptor. $^{35}$ Here, we analyze this SWCNT-based Type-I heterojunction by femtosecond TA spectroscopy. A comparison with surfactant-dispersed $(6,5)$ SWCNTs in water (hereafter referred to as SWCNT), which allows for observations of exciton dynamics without energy or charge transfer, ${ }^{37-40}$ is conducted throughout this work. The exciton dynamics of the Hybrid in tetrahydrofuran and SWCNT in water are investigated upon the $\mathrm{E}_{11}, \mathrm{E}_{22}$, and $\mathrm{E}_{33}$ excitation with variable excitation fluences, and PCT products in the Hybrid are analyzed. We demonstrate that the PCT from photoexcited $(6,5)$ SWCNT to PFO-BPy is driven by the energetically favorable $\mathrm{E}_{33}$ excitation and may be also driven by the energetically unfavorable $\mathrm{E}_{22}$ excitation. The latter process occurs via Auger recombination of excitons and charge carriers at high excitation fluences.

\section{EXPERIMENTAL SECTION}

Surfactant-Based Carbon Nanotube Dispersion and Chirality Enrichment. The preparation of aqueous $(6,5)$ suspension is based on the $\mathrm{pH}$-modulated aqueous two-phase extraction (ATPE) method. $^{41}$ Simply, a $20 \mathrm{mg}$ portion of CoMoCAT SG65i SWCNTs powder (SouthWest Nanotechnologies, lot no. SG65i-L58) was suspended in $20 \mathrm{~mL}$ of aqueous $1 \%(\mathrm{~m} / \mathrm{v})$ DOC (BioChemica) by tip sonication (Weber Ultrasonics, $35 \mathrm{kHz}, 16 \mathrm{~W}$ in continuous mode) for 1 $\mathrm{h}$ while immersed in an ice bath. The resulting dispersion was centrifuged at $45560 \mathrm{~g}$ (Beckman Optima L-80 XP, SW $40 \mathrm{Ti}$ rotor) for $1 \mathrm{~h}$, and the supernatant collected for ATPE. ATPE then was performed at a concentration of $4 \%(\mathrm{~m} / \mathrm{m})$ dextran (Mw $70000 \mathrm{Da}, \mathrm{TCI}), 8 \%$ (m/m) PEG (Mw $6000 \mathrm{Da}$, Alfa Aesar) with $0.5 \% \mathrm{~m} / \mathrm{v}$ SDS (Sigma-Aldrich), and $0.05 \% \mathrm{~m} / \mathrm{v}$ DOC (Sigma-Aldrich). First, $16 \mu \mathrm{L}$ of $\mathrm{HCl}(0.5 \mathrm{M})$ was added to a $16 \mathrm{~mL}$ ATPE system (with $0.8 \mathrm{~mL}$ of SWCNT suspension) to remove the nanotubes with a diameter larger than the $(6,5)$. Next, a fresh mimic top phase was added along with some new $\mathrm{HCl}(20 \mu \mathrm{L})$ to obtain $(6,5)$ on the top phase. Finally, the $(6,5)$ enriched top phase was added to a fresh mimic bottom phase with $40 \mu \mathrm{L}$ of sodium hypochlorite ( $\mathrm{NaClO}$, Honeywell) and $20 \mu \mathrm{L}$ of compensated $\mathrm{HCl}$ to separate the metallic tubes with similar diameters. After centrifugation, the purified single chirality $(6,5)$ remained in the bottom phase while the metallic tubes $(7,4)$ partitioned to the top phase.

Carbon Nanotube Dispersion by Polymer Wrapping. As described previously, ${ }^{2}$ nearly monochiral polymer-wrapped $(6,5)$ SWCNTs were obtained by shear force mixing (Silverson L2/Air, $10230 \mathrm{rpm}, 72 \mathrm{~h}$ ) of CoMoCAT raw material (Chasm Advanced Materials, SG65i-L58, $\left.0.38 \mathrm{~g} \mathrm{~L}^{-1}\right)$ and poly-[(9,9dioctylfluorenyl-2,7-diyl)-alt-(6,6')-(2,2'-bipyridine)] (PFOBPy, American Dye Source, Mw $40000 \mathrm{Da}, 0.5 \mathrm{~g} \mathrm{~L}^{-1}$ ) in toluene. Aggregates were removed by centrifugation at $60000 \mathrm{~g}$ (Beckman Coulter Avanti J26XP centrifuge) for $2 \times 45 \mathrm{~min}$ with intermediate supernatant extraction and final filtration through a poly(tetrafluoroethylene) (PTFE) syringe filter (5 $\mu \mathrm{m}$ pore size). To remove unbound $\mathrm{PFO}-\mathrm{BPy}$, the dispersion was passed through a PTFE membrane filter (Merck Millipore, JVWP, $0.1 \mu \mathrm{m}$ pore size) to collect the SWCNTs and separate the unbound polymer. The SWCNT-coated membrane was further washed by immersion in toluene at $80{ }^{\circ} \mathrm{C}$ for $10 \mathrm{~min}$. Finally, the washed, PFO-BPy-wrapped $(6,5)$ SWCNTs (Hybrid) were redispersed from the membrane by bath sonication in a small volume of tetrahydrofuran (THF) for 30 $\min$.

Stationary and Transient Absorption Spectral Measurements. Stationary UV-visible-NIR absorption spectra were measured on a V-770 (JASCO) spectrophotometer. Femtosecond transient absorption (TA) spectral measurements were performed on a commercial TA spectrograph (Helios Fire, Ultrafast Systems). The pump spectra centered at 1000,576 , or $350 \mathrm{~nm}$ were generated with a commercial optical parametric amplifier (TOPAS-Prime, Light Conversion), which was pumped by a regeneratively amplified femtosecond Ti:sapphire laser (Astrella, Coherent) centered at $800 \mathrm{~nm}$, with a $4 \mathrm{kHz}$ repetition rate, $78 \mathrm{fs}$ pulse durations, and $1.6 \mathrm{~mJ}$ pulse energy. The spectra of pump pulses are shown in Figure S1 in the Supporting Information (SI). The spot size of the focused pump beam was about $250 \mu \mathrm{m}$. Typically, pump fluences were $200 \mu \mathrm{J} \cdot \mathrm{cm}^{-2}$ for pulse energies of $100 \mathrm{~nJ}$. The supercontinuum probe beam was generated in a sapphire substrate for the NIR detection $(800-1350 \mathrm{~nm})$ or in a calcium fluoride substrate for the UV-vis detection (330$650 \mathrm{~nm})$. The pump beam was linearly polarized at the magic angle $\left(54.7^{\circ}\right)$ relative to the probe beam. TA spectra were corrected for the group velocity dispersion of the broad-band probe beam before analysis. All measurements were performed under ambient conditions.

Spectroelectrochemical Measurement. The spectroelectrochemical measurement was carried out on a Lambda 750 (PerkinElmer) UV-visible spectrophotometer combined with a $\mathrm{CHI}$ 660D ( $\mathrm{CH}$ Instruments) potentiostat. The working electrode was an ITO glass $(<10 \Omega$ /square $)$. The counter 
electrode was a platinum coil. The reference electrode was $\mathrm{Ag} /$ $\mathrm{AgCl}$. The electrolyte is $0.1 \mathrm{M} n \mathrm{Bu}_{4} \mathrm{NPF}_{6}$. Experiments were carried out at ambient temperature under the protection of nitrogen.

\section{RESULTS}

Stationary Spectral Characterizations. The stationary absorption spectra (Figure 2) of SWCNT/PFO-BPy Hybrid

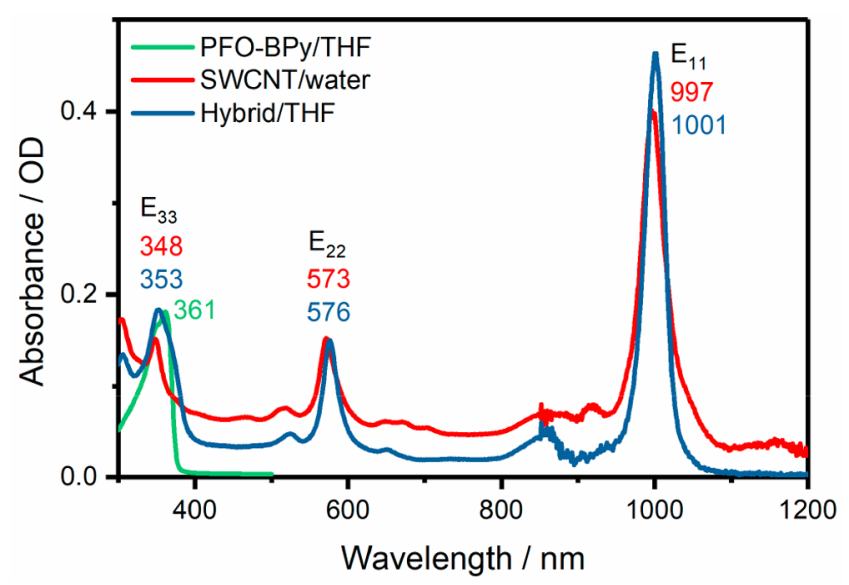

Figure 2. Stationary absorption spectra of surfactant-dispersed $(6,5)$ SWCNT in water, PFO-BPy-wrapped $(6,5)$ SWCNT Hybrid in THF, and PFO-BPy in THF. The positions of absorption peaks are marked with corresponding colors.

and surfactant-dispersed SWCNT show the typical excitonic transitions, i.e., $\mathrm{E}_{00} \rightarrow \mathrm{E}_{11}, \mathrm{E}_{00} \rightarrow \mathrm{E}_{22}$, and $\mathrm{E}_{00} \rightarrow \mathrm{E}_{33}\left(\mathrm{E}_{00}\right.$ denotes the ground state in the exciton picture, as shown in Figure S1 in the Supporting Information), peaking around 1000,576 , and $350 \mathrm{~nm}$, respectively. The absorption of PFOBPy in the Hybrid system is observed as a shoulder on the red side of the $E_{00} \rightarrow E_{33}$ absorption band (see Supporting Information, Figure S2 for details). The visibility of the $\mathrm{E}_{00} \rightarrow$ $\mathrm{E}_{33}$ absorption is due to the carefully reduced PFO-BPy concentration. Moreover, the chirality distributions of the SWCNT and Hybrid samples have been characterized by absorption spectroscopy (see Supporting Information, section B). ${ }^{42}$

Transient Absorption Spectra of the SWCNT. Figure 3 displays selected near-infrared (NIR) TA spectra of SWCNT upon the $E_{11}, E_{22}$, and $E_{33}$ resonant excitations. Due to the purity of the sorted $(6,5)$ SWCNT, several known absorption features are clearly observed in the TA spectra. Upon $E_{11}$ excitation (Figure $3 \mathrm{a}$ ) the dominant negative signal centered at $\sim 1000 \mathrm{~nm}$ arises from the $\mathrm{E}_{00} \rightarrow \mathrm{E}_{11}$ bleach, as reported previously. ${ }^{43}$ The photoinduced absorption (PA) band centered at $\sim 1110 \mathrm{~nm}(\sim 1.12 \mathrm{eV})$ on the red side of the $\mathrm{E}_{00} \rightarrow \mathrm{E}_{11}$ bleach builds up within the instrumental response time and decays subsequently. This PA band has been assigned to the transition from exciton to biexciton $\left(\mathrm{E}_{11} \rightarrow \mathrm{E}_{11, \mathrm{BX}}\right)^{39,44}$ Another PA band peaking at $\sim 1143 \mathrm{~nm}(\sim 1.08 \mathrm{eV})$ is evident after $\sim 10$ ps and dominating the long-time scale spectra up to the limit of the measurement time window. Previous studies attributed this band to the triplet exciton absorption $\left({ }^{3} \mathrm{E}_{11} \rightarrow\right.$ $\left.{ }^{3} \mathrm{E}_{\mathrm{nn}}\right){ }^{45}$ Additionally, a broad PA band over $1200-1350 \mathrm{~nm}$ decays rapidly within the initial $\sim 1$ ps. This spectral feature is commonly observed for the optical excitation into the $E_{11}$ excitonic band at high pump fluence and has been discussed to

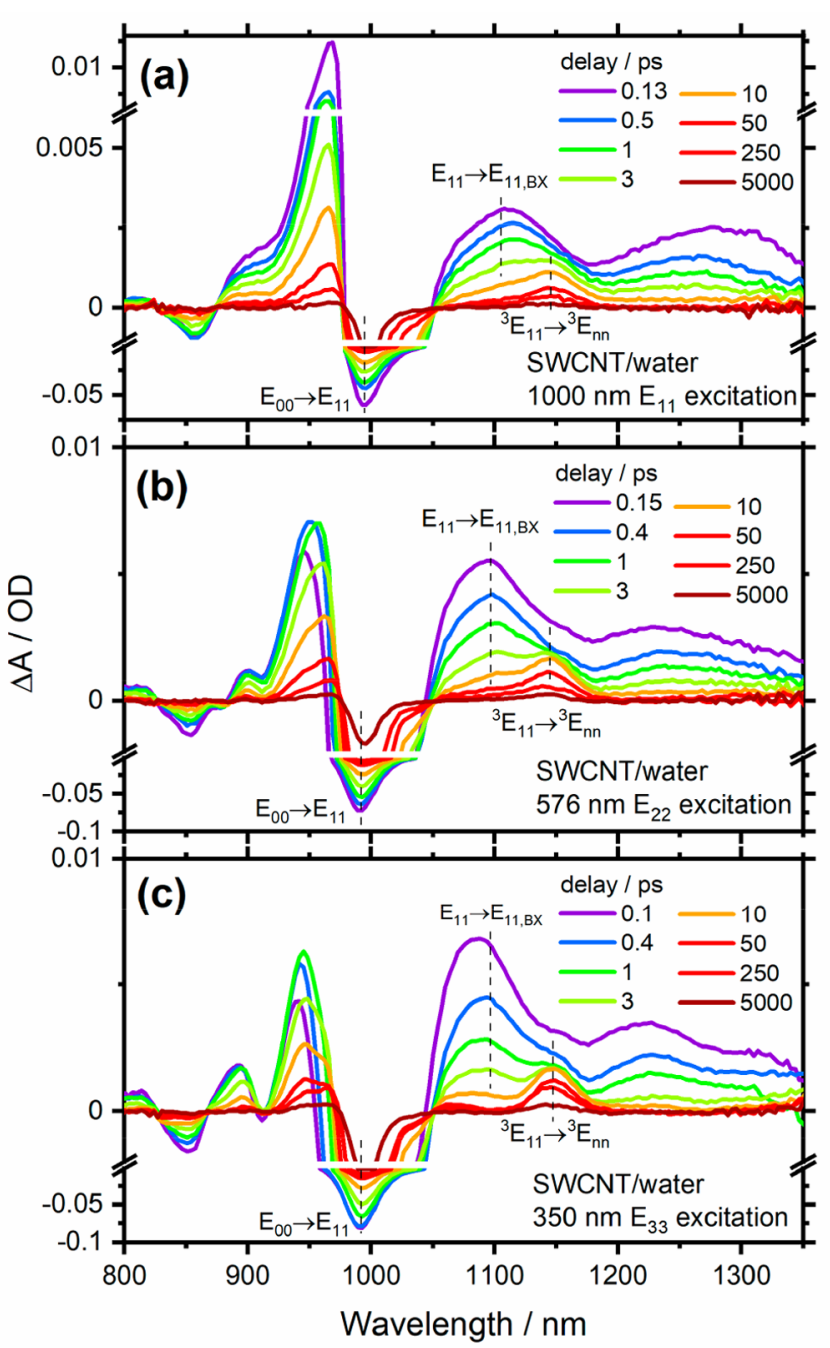

Figure 3. Selected TA spectra of SWCNT in water upon the (a) $E_{11}$, (b) $\mathrm{E}_{22}$, and (c) $\mathrm{E}_{33}$ excitations. Experimental conditions: (a) $\lambda_{\mathrm{ex}}=$ $1000 \mathrm{~nm}$, (b) $\lambda_{\text {ex }}=576 \mathrm{~nm}$, and (c) $\lambda_{\text {ex }}=350 \mathrm{~nm}$; pump energy: 100 $\mathrm{nJ} \cdot$ pulse $^{-1}$. Dotted lines highlight major transition manifolds.

be related to multiple $\mathrm{E}_{11}$ exciton interactions. ${ }^{38,39,46,47}$ The PA band on the blue side of the $\mathrm{E}_{00} \rightarrow \mathrm{E}_{11}$ bleach is attributed to a transition from a dark $\mathrm{E}_{11}$ state to the unbound two-exciton manifold. $^{48}$

Resonant excitation into the $\mathrm{E}_{22}$ or $\mathrm{E}_{33}$ excitonic band of SWCNT results basically in the same spectral features (Figure $3 \mathrm{~b}, \mathrm{c})$ : the $\mathrm{E}_{11} \rightarrow \mathrm{E}_{11, \mathrm{BX}}$ and ${ }^{3} \mathrm{E}_{11} \rightarrow{ }^{3} \mathrm{E}_{\mathrm{nn}}$ transitions are still evident and show spectral line shapes and positions in agreement with those upon the $\mathrm{E}_{11}$ excitation (vide supra). For excitons created by higher band transitions, such as $E_{22}$, the intersubband relaxation to the $\mathrm{E}_{11}$ state has been shown to take place within $100 \mathrm{fs}^{30,40,48,49}$ Upon $\mathrm{E}_{22}$ or $\mathrm{E}_{33}$ excitation, the $\mathrm{E}_{00} \rightarrow \mathrm{E}_{11}$ bleach with a large negative amplitude becomes broader and slightly blue-shifted, which results in larger overlap with the blue-side PA band. Similar spectral dynamics of SWCNT are observed in our results upon the excitation of either the $\mathrm{E}_{11}, \mathrm{E}_{22}$, or $\mathrm{E}_{33}$ excitonic band. ${ }^{44}$

Transient Absorption Spectra of the SWCNT/PFOBPy Hybrid. In the previous section, the intrinsic exciton dynamics of $(6,5)$ SWCNTs upon different excitonictransition excitations have been shown. Our focus now turns to the excited-state dynamics of the $(6,5)$ SWCNT/PFO-BPy 
formed heterojunction to find evidence for PCT. Selected NIR TA spectra of the Hybrid upon the $\mathrm{E}_{11}, \mathrm{E}_{22}$, and $\mathrm{E}_{33}$ excitations are shown in Figure 4. Besides the known $\mathrm{E}_{11} \rightarrow \mathrm{E}_{11, \mathrm{BX}}(\sim 1100$

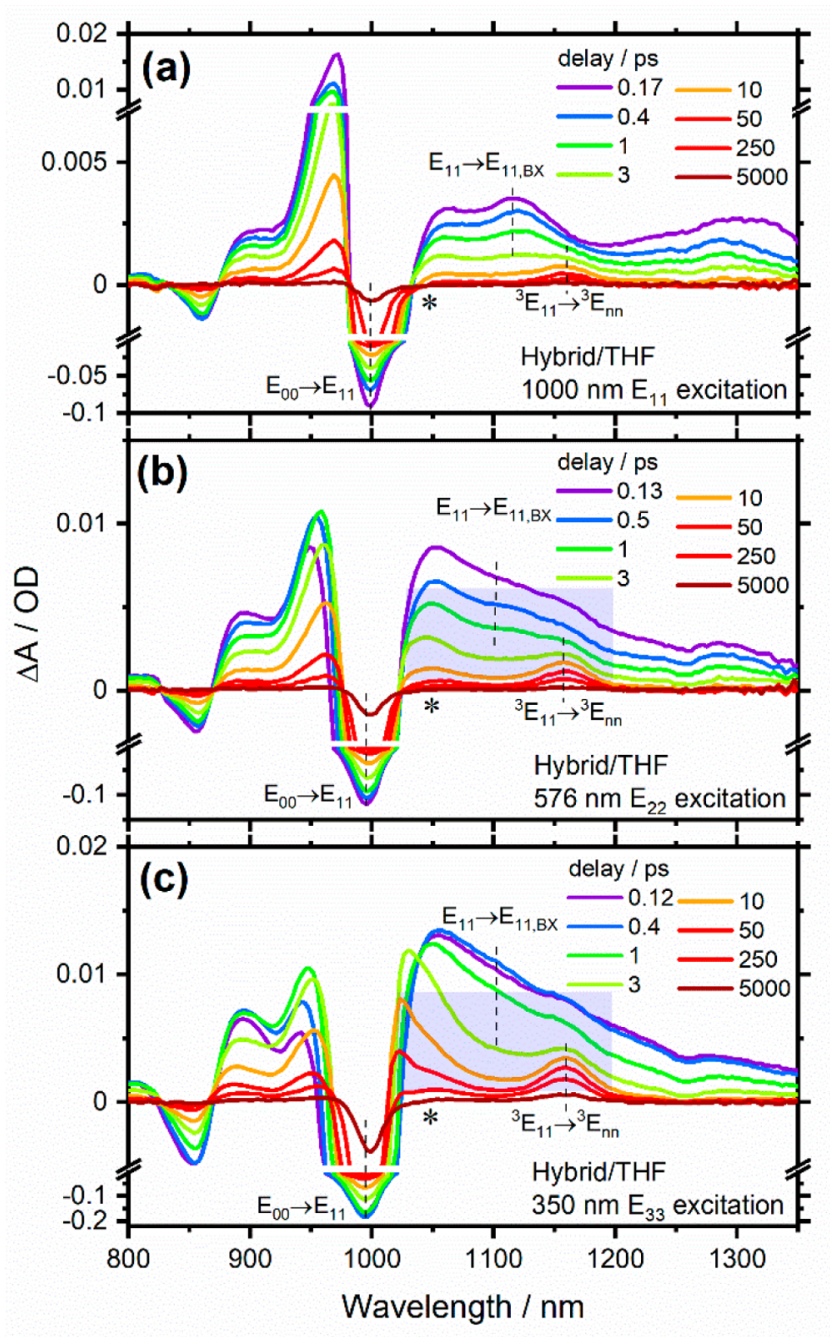

Figure 4. Selected TA spectra for the Hybrid in THF upon the (a) $\mathrm{E}_{11}$, (b) $\mathrm{E}_{22}$, and (c) $\mathrm{E}_{33}$ excitations. Experimental conditions: (a) $\lambda_{\mathrm{ex}}$ $=1000 \mathrm{~nm}$, (b) $\lambda_{\mathrm{ex}}=576 \mathrm{~nm}$, and (c) $\lambda_{\mathrm{ex}}=350 \mathrm{~nm}$; pump energy: $100 \mathrm{~nJ} \cdot$ pulse $^{-1}$. Dotted lines highlight major transition manifolds. The shaded shapes indicate the absorption signature of the suspected SWCNT polaron. The asterisks $(*)$ denote the wavelength of 1050 $\mathrm{nm}$.

$\mathrm{nm}, \sim 1.13 \mathrm{eV})$ and ${ }^{3} \mathrm{E}_{11} \rightarrow{ }^{3} \mathrm{E}_{\mathrm{nn}}(\sim 1160 \mathrm{~nm}, \sim 1.07 \mathrm{eV})$ transitions, an additional broad PA band emerges on the red side of the $E_{00} \rightarrow E_{11}$ bleach in the Hybrid upon $E_{22}$ and $E_{33}$ excitation (highlighted by shaded areas in Figure $4 \mathrm{~b}, \mathrm{c}$ ). It covers the spectral range of $\sim 1050-1200 \mathrm{~nm}$ that overlaps with the absorption of $\mathrm{E}_{11} \rightarrow \mathrm{E}_{11, \mathrm{BX}}$ and ${ }^{3} \mathrm{E}_{11} \rightarrow{ }^{3} \mathrm{E}_{\mathrm{nn}}$ transitions and lasts for tens of picoseconds. Furthermore, on this time scale, we note that the $\mathrm{E}_{00} \rightarrow \mathrm{E}_{11}$ bleach in the TA spectra of the Hybrid upon the $\mathrm{E}_{33}$ excitation manifests a dynamic blueshift of up to $\sim 5 \mathrm{~nm}$. We summarize the peak dynamics in Figure 5. The time-dependent peak-shifting of the $\mathrm{E}_{00} \rightarrow \mathrm{E}_{11}$ bleach was extracted from the TA spectra by Gaussian-peak fitting in the energy domain within the spectral region of the dominant bleach band centered at $\sim 1000 \mathrm{~nm}$. Considering that the exciton density is strongly dependent on the excitation fluence (section I), we investigated this peak shift for the SWCNT and Hybrid with various excitation fluences (see Supporting Information, section C for fluence-dependent TA spectra).

As shown in Figure 5a, the peak position of the $\mathrm{E}_{00} \rightarrow \mathrm{E}_{11}$ bleach for the SWCNT upon the $\mathrm{E}_{11}$ excitation remains stable across the investigated time window. For the SWCNT upon $\mathrm{E}_{22}$ or $\mathrm{E}_{33}$ excitation (Figure $5 \mathrm{~b}$ and $5 \mathrm{c}$, respectively), the bleach peak shows a slight and smooth red-shift through the entire time window. This trend is widely observed when tracking the dynamics of excitonic band bleach recovery in SWCNTs, and it can be explained by intersubband and intrasubband relaxation. ${ }^{39,50}$ Upon exciting the Hybrid with different pump wavelengths, the initial peak position at $\sim 0.1 \mathrm{ps}$ shifts slightly, which matches well with the trend observed in the SWCNT. However, the peak-shifting dynamics are significantly different for the Hybrid. Besides the smooth red-shift through the entire time window, a dynamic blue-shift of the $\mathrm{E}_{00} \rightarrow \mathrm{E}_{11}$ bleach is particularly evident within the time delay from 1 to $50 \mathrm{ps}$ when the Hybrid is pumped at $E_{33}$ (Figure 5f). Notably, the extent of the dynamic blue-shift progressively becomes larger with increasing excitation fluence. When the peak-shifting curves of the Hybrid upon $E_{22}$ excitation (Figure 5e) are examined, although these curves do not show an obvious blue-shift as $\mathrm{E}_{33}$ excitation, the peak position plateaus on the same time scale at the highest excitation energy. For the Hybrid upon $E_{11}$ excitation, we hardly observe this trend (Figure 5d), and it behaves almost the same as the SWCNT upon $\mathrm{E}_{11}$ excitation. The additional peak dynamics of the $\mathrm{E}_{00} \rightarrow \mathrm{E}_{11}$ bleach in the Hybrid upon $\mathrm{E}_{33}$ (and less pronounced upon $\mathrm{E}_{22}$ ) excitation indicate an additional quasiparticle with a blue-shifted $\mathrm{E}_{00} \rightarrow \mathrm{E}_{11}$ bleach and an observable buildup time, which may be the PCT product. One point to note is that the dynamic blue-shift in tens of picoseconds does not directly reflect real population dynamics. It only suggests that the share of this new species is increasing among all quasiparticles, and the population of each transient species may be decaying individually on that time scale.

Absorption Features of Charge Transfer Products. To identify the PCT in the Hybrid, we examine the spectroscopic features of potential PCT transient products. ${ }^{22,51}$ Charged transient products in SWCNTs have been demonstrated to be two kinds of quasiparticles: (a) the polaron, which describes a conduction electron (or hole) strongly coupled with the lattice ions, ${ }^{52}$ or (b) the trion, which is a three-body charge-exciton bound state. $38,47,53,54$

The $(6,5)$ SWCNT polaron can be created in steady state by two methods, namely redox-chemical doping ${ }^{22,25,55-57}$ and electrochemical doping. ${ }^{6,58,59}$ We carried out the redoxchemical hole-doping of the Hybrid dispersion with $\mathrm{NOBF}_{4}$ $\left(+1.00 \mathrm{~V} \text { vs Fc/ } \mathrm{Fc}^{+} \text {in } \mathrm{CH}_{2} \mathrm{Cl}_{2}\right)^{60}$ as a one-electron oxidant. ${ }^{61}$ As shown in Figure 6a, along with the increasing doping level, the stationary absorption of the $\mathrm{E}_{00} \rightarrow \mathrm{E}_{11}$ transition $(\sim 1000$ $\mathrm{nm}$ ) decreases dramatically and exhibits a blue-shift in wavelength. On the red side of the dominant $E_{00} \rightarrow E_{11}$ transition, stationary spectra of the doped Hybrid feature a broad absorptive band extending from 1030 to $1200 \mathrm{~nm}$, including a peak around $\sim 1150 \mathrm{~nm}$. The weak absorption at $1150 \mathrm{~nm}$ before the oxidative titration $\left(\left[\mathrm{NOBF}_{4}\right]=0 \mu \mathrm{M}\right)$ results from the slight p-doping of SWCNTs in the air. ${ }^{62} \mathrm{We}$ expect that the polymer in the Hybrid remains unoxidized during the titration, since the $\mathrm{HOMO}$ energy of $\mathrm{PFO}-\mathrm{BPy}$ is 


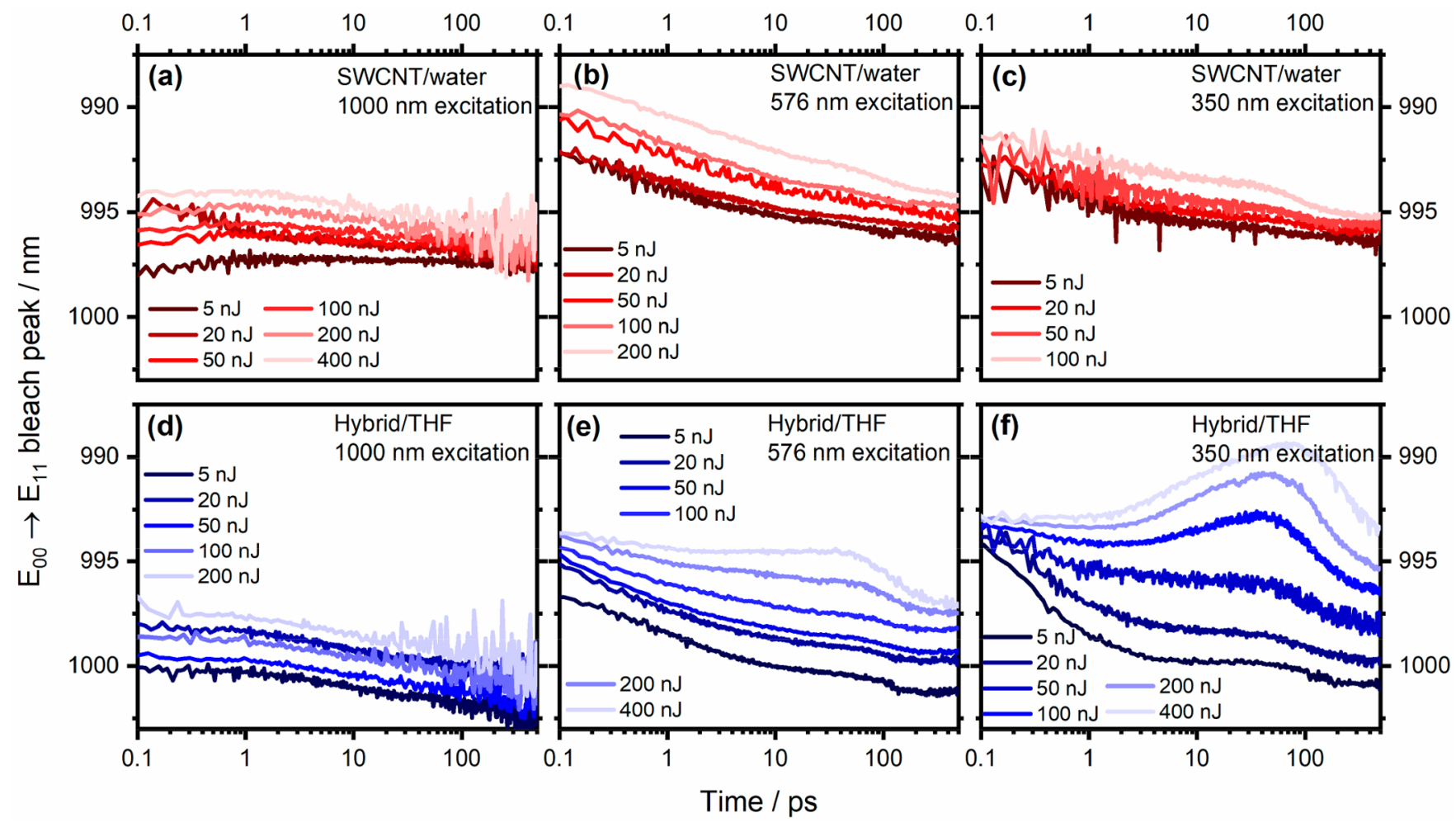

Figure 5. Pump-energy-dependent peak-shifting dynamics of the $\mathrm{E}_{00} \rightarrow \mathrm{E}_{11}$ bleaching in TA spectra of the SWCNT (a, b, c) and the Hybrid (d, e, f) in the time window of 0.1-500 ps. Excitation wavelength and corresponding pump energy per pulse are given in legends. Due to dispersion instability under high pump fluences, TA spectra of SWCNT are unavailable at higher fluences in b and c.

stabilized by $1.4 \mathrm{eV}$ with respect to the first valence band of $(6,5)$ SWCNT. The previously reported electrochemical doping for the Hybrid in films shows a very similar trend to the redox doping. ${ }^{6}$ Note that for very high doping levels, the entire NIR absorption of the Hybrid is strongly bleached. We conclude that the above spectral features describe the $(6,5)$ SWCNT hole-polaron absorption transition, denoted as $\mathrm{E}_{00}^{+}$ $\rightarrow \mathrm{E}^{+}{ }_{11}$.

In order to examine the spectral signature of trions, we conducted the TA measurement on the heavily hole-doped $\left(\left[\mathrm{NOBF}_{4}\right] \sim 128 \mu \mathrm{M}\right)$ Hybrid under $\mathrm{E}_{11}$ excitation. ${ }^{47}$ As shown in Figure $6 \mathrm{~b}$, the initial TA spectra show the $\mathrm{E}_{00} \rightarrow \mathrm{E}_{11}$ bleach at $\sim 1000 \mathrm{~nm}$ and $\mathrm{E}^{+}{ }_{00} \rightarrow \mathrm{E}^{+}{ }_{11}$ bleach at $\sim 1150 \mathrm{~nm}$. The $\mathrm{E}^{+}{ }_{00} \rightarrow \mathrm{E}^{+}{ }_{11}$ bleach decreases within $\sim 1 \mathrm{ps}$, and meanwhile a new absorptive species centered at $\sim 1190 \mathrm{~nm}(\sim 1.04 \mathrm{eV})$ is formed. This band has been assigned to a positive trion $\left(\mathrm{Tr}^{+}{ }_{11}\right.$ $\left.\rightarrow \mathrm{Tr}^{+}{ }_{n n}\right)$ absorption of $(6,5)$ SWCNTs. ${ }^{38,47}$ The spectral signature of trions in the Hybrid is consistent with that observed in $(6,5)$ SWCNT superstructures reported by Therien et al. ${ }^{38,47}$

\section{DISCUSSION}

Observation of Charge Transfer in SWCNT/PFO-BPy Hybrid. Combining the results of redox-chemical and electrochemical doping (vide supra), we can summarize the spectroscopic features of the $(6,5)$ SWCNT hole-polaron $\left(\mathrm{E}^{+}{ }_{00}\right.$ $\left.\rightarrow \mathrm{E}^{+}{ }_{11}\right)$ in comparison with the excitonic absorption spectrum of neutral $(6,5)$ SWCNTs. The $(6,5)$ SWCNTs hole-polaron features a bleached and blue-shifted $\mathrm{E}_{00} \rightarrow \mathrm{E}_{11}$ transition and an additional absorption band covering 1030-1200 nm, the line shape of which strongly depends on the charge carrier level. ${ }^{25,55,57-59,63}$ The electronic absorption transitions of oxidized nanotubes are explained by the electron depletion of the top of the valence band, which results in an increase in the $\mathrm{E}_{00} \rightarrow \mathrm{E}_{11}$ transition energy and leads to additional electronic transitions. ${ }^{25,59}$ It is noteworthy that studies by electron paramagnetic resonance (EPR) spectroscopy reveal that the unpaired electrons in lightly reduced SWCNTs are relatively free and fast-relaxing. ${ }^{28}$ This is certainly valid for metallic SWCNTs but has been corroborated as well for semiconducting SWCNTs. ${ }^{64}$ Thus, the "polaron" in SWCNTs is relatively delocalized, which may prolong the lifetime of the charge-separation state.

As shown in Figure 5, TA spectra of the Hybrid feature a dynamic blue-shift of the $\mathrm{E}_{00} \rightarrow \mathrm{E}_{11}$ bleach in picoseconds following the $E_{22}$ and $E_{33}$ excitations, which strongly suggests the formation of the SWCNT polaron $\left(\mathrm{E}^{ \pm}{ }_{00} \rightarrow \mathrm{E}^{ \pm}{ }_{11}\right)$. Although the triplet absorption also contributes to the absorption around $1160 \mathrm{~nm}$ where an absorption peak of $\mathrm{E}^{ \pm}{ }_{00} \rightarrow \mathrm{E}^{ \pm}{ }_{11}$ is located, the TA spectra of SWCNT indicate that the formation of triplet excitons is independent of the excitation energy and fluence. According to the reported time constant of intersystem crossing of $\sim 20$ ps in $(6,5)$ SWCNTs, ${ }^{45}$ the broad absorption band at 1030-1200 nm and the peak around $1160 \mathrm{~nm}$ formed in the first few picoseconds in the Hybrid upon high-energy excitation should be attributed to the $\mathrm{E}^{ \pm}{ }_{00} \rightarrow \mathrm{E}^{ \pm}{ }_{11}$ transition. However, the spectral overlap between $\mathrm{E}^{ \pm}{ }_{00} \rightarrow \mathrm{E}^{ \pm}{ }_{11}, \mathrm{E}_{11} \rightarrow \mathrm{E}_{11, \mathrm{BX}}$, and ${ }^{3} \mathrm{E}_{11}$ $\rightarrow{ }^{3} \mathrm{E}_{\mathrm{nn}}$ transitions (compare Figure 4 with Figure 6a) impedes a spectral disentanglement of the SWCNT polaron dynamics via global analysis of the TA dynamics of the Hybrid. Thus, we select the TA kinetics at $1050 \mathrm{~nm}$, where an isosbestic point with almost zero $\Delta A$ is located in the TA spectra of the SWCNT, to further investigate the formation of the SWCNT 


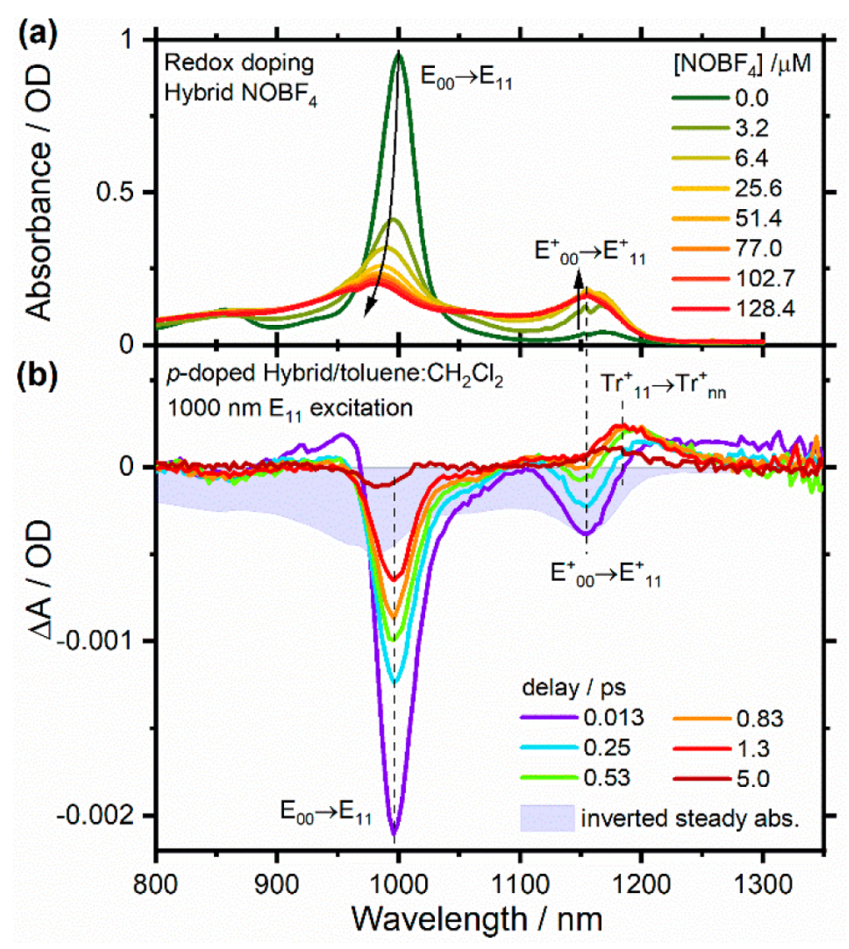

Figure 6. (a) NIR stationary absorption spectra monitor the oxidative titration of the Hybrid with $\mathrm{NOBF}_{4}$ in toluene: $\mathrm{CH}_{2} \mathrm{Cl}_{2}$ (ratio 1:1) mixed solution. Experimental conditions: $[(6,5)$ SWCNT $] \sim 2.74$ $\mathrm{nM}$; SWCNT length $\sim 1000 \mathrm{~nm}$; optical path length $=10 \mathrm{~mm}$. (b) Selected TA spectra for a heavily hole-doped $\left(\left[\mathrm{NOBF}_{4}\right] \sim 128 \mu \mathrm{M}\right)$ Hybrid in toluene: $\mathrm{CH}_{2} \mathrm{Cl}_{2}$ (ratio 1:1) mixed solution. Experimental conditions: $\lambda_{\mathrm{ex}}=1000 \mathrm{~nm}$, i.e., in resonance with $\mathrm{E}_{11}$; pump energy $=$ $50 \mathrm{~nJ} \cdot$ pulse $^{-1}$. Scaled steady-state absorption spectrum (inverted shaded shape) is shown for comparison.

polaron in the Hybrid. As shown in Figure $7 a-c$, the normalized kinetic traces for the Hybrid pumped at very low pump energy ( 5 or $20 \mathrm{~nJ} \cdot$ puls $^{-1}$ ) show a smooth decay which is generally the same for the $E_{11}, E_{22}$, and $E_{33}$ excitations. This decay component arises from the absorption edge of $\mathrm{E}_{11} \rightarrow$ $\mathrm{E}_{11, \mathrm{BX}}$ (centered at $1100 \mathrm{~nm}$ ) transition. When the pump energy is increased from 50 to $400 \mathrm{~nJ} \cdot \mathrm{pulse}^{-1}$, the dynamics of the SWCNT polaron emerge. It becomes progressively evident in the Hybrid upon $E_{22}$ and $E_{33}$ excitation (Figure $7 b, c$ ) and overlaps with the $\mathrm{E}_{11} \rightarrow \mathrm{E}_{11, \mathrm{BX}}$ absorption. The absorption amplitude reaches its maximum at $\sim 3$ ps and slowly decays over a few hundred picoseconds. The spectroscopic features of SWCNT polaron absorption over 1050-1200 $\mathrm{nm}$ can be observed in the normalized TA spectra of the Hybrid at the probe delay of $3 \mathrm{ps}$ (Figure $7 \mathrm{e}, \mathrm{f}$ ), matching well with the absorptive feature of the SWCNT hole-polaron $\left(\mathrm{E}^{+}{ }_{00} \rightarrow \mathrm{E}^{+}{ }_{11}\right.$, shown in blue lines) obtained by chemical doping (vide supra). In the TA spectra of the Hybrid upon $\mathrm{E}_{11}$ excitation, the signature of the SWCNT polaron is hardly observed in the respective kinetics and spectra (Figure $7 \mathrm{a}, \mathrm{d}$ ). In addition, using the spectroelectrochemical method, the PFO-BPy polaron was prepared and observed in the steady-state spectrum. As one would expect, the formation of the PFO$\mathrm{BPy}$ polaron is also observed in the UV region of the Hybrid TA spectra around $380 \mathrm{~nm}$ (see Supporting Information, sections $\mathrm{E}$ and $\mathrm{F}$ ). Hence, we conclude that a $\mathrm{PCT}$ reaction takes place in the SWCNT/PFO-BPy Hybrid system, forming an interfacial charge-separated state. ${ }^{22}$
It is worth adding that we have not observed any spectral evidence of trion formation, as previously identified from the hole-doped Hybrid solution (Figure 6b), in the TA spectra of undoped SWCNT or Hybrid.

In the Hybrid, the direction of PCT can be deduced by analyzing the excitation distribution between the two components. In the case of $\mathrm{E}_{11}$ or $\mathrm{E}_{22}$ excitation of the Hybrid, the pump pulse centered at 1000 or $576 \mathrm{~nm}$ is far away from the UV resonant absorption of PFO-BPy. Thus, the PCT in both cases purely originates from the excited $(6,5)$ SWCNT to the PFO-BPy. In the case of $\mathrm{E}_{33}$ excitation, the pump spectrum centered at $350 \mathrm{~nm}$ is resonant with $(6,5)$ SWCNT as well as with PFO-BPy. Since PCT from excited PFO-BPy to the $(6,5)$ SWCNTs is also energetically favorable in this case, one needs to evaluate the excitation contribution from $(6,5)$ SWCNT and PFO-BPy in the Hybrid by comparing the initial UV-vis TA spectra of the Hybrid, SWCNT, and PFO-BPy excited at $350 \mathrm{~nm}$. As shown in Figure 8 , the TA spectrum of $\mathrm{PFO}-\mathrm{BPy}$ at 0.2 ps clearly manifests a negative bleach peak $(\sim 365 \mathrm{~nm})$ and a negative stimulated emission peak $(\sim 390 \mathrm{~nm})$ (see Supporting Information, section D for the TA spectral analysis of PFO$\mathrm{BPy})$. However, the above TA spectral features from excited PFO-BPy are hardly observed in the TA spectrum of the Hybrid at the same time delay (see Supporting Information, section $\mathrm{E}$ for a comparison of the full dynamics). The Hybrid TA spectrum only retains the excitation features of the SWCNT, especially for the region of $350-450 \mathrm{~nm}$, and the excitation contribution of $\mathrm{PFO}-\mathrm{BPy}$ is minimal in comparison to the excitation of $(6,5)$ SWCNTs in the Hybrid. Thus, we conclude that although the PCT from excited PFO-BPy to $(6,5)$ SWCNT cannot be completely ruled out, the current analysis shows that, in the case of $\mathrm{E}_{33}(350 \mathrm{~nm})$ excitation of the Hybrid, the PCT from excited $(6,5)$ SWCNT to PFO$\mathrm{BPy}$ is absolutely dominant.

An additional point to address is whether an electron or hole transfer takes place. Since the electron and hole in SWCNT have a similar effective mass, electron- and hole-polaron will show very similar absorptive signatures at the same doping level. ${ }^{65}$ However, the energy level alignment in the Hybrid (Figure 1) implies that the electron transfer from the third conduction subband $\left(c_{3}\right)$ of $(6,5)$ SWCNT to the LUMO of $\mathrm{PFO}-\mathrm{BPy}$ has a larger driving force than the hole transfer from the third valence subband $\left(v_{3}\right)$ to the HOMO. Therefore, photoinduced electron transfer from $(6,5)$ SWCNT to PFOBPy is more likely to take place than hole transfer.

Charge Transfer Assisted by Auger Recombination. As discussed above, when the wide-bandgap PFO-BPy acts as a charge acceptor in the Hybrid, the PCT from the $E_{11} / E_{22^{-}}$ excited $(6,5)$ SWCNT to PFO-BPy is energetically unfavorable (Figure 1). However, when multiple excitons are present on a SWCNT in a high-excitation-density regime, strong exciton-exciton interactions lead to Auger recombination of excitons, also known as exciton-exciton annihilation (EEA), in which one exciton recombines to the ground state and the other is promoted to a higher excitonic state or dissociates into free charge carriers. ${ }^{34,35,39,66}$ Hence, Auger recombination of excitons can be regarded as a potential energy up-conversion process that forms one high-energy exciton through absorbing two low-energy photons. Under this assumption, the occurrence of PCT driven by $\mathrm{E}_{22}(576 \mathrm{~nm}, 2.15 \mathrm{eV})$ excitation in the Hybrid indicates that $\mathrm{E}_{33}(350 \mathrm{~nm}, 3.54 \mathrm{eV})$ or higherenergy excitons are generated via Auger recombination of 

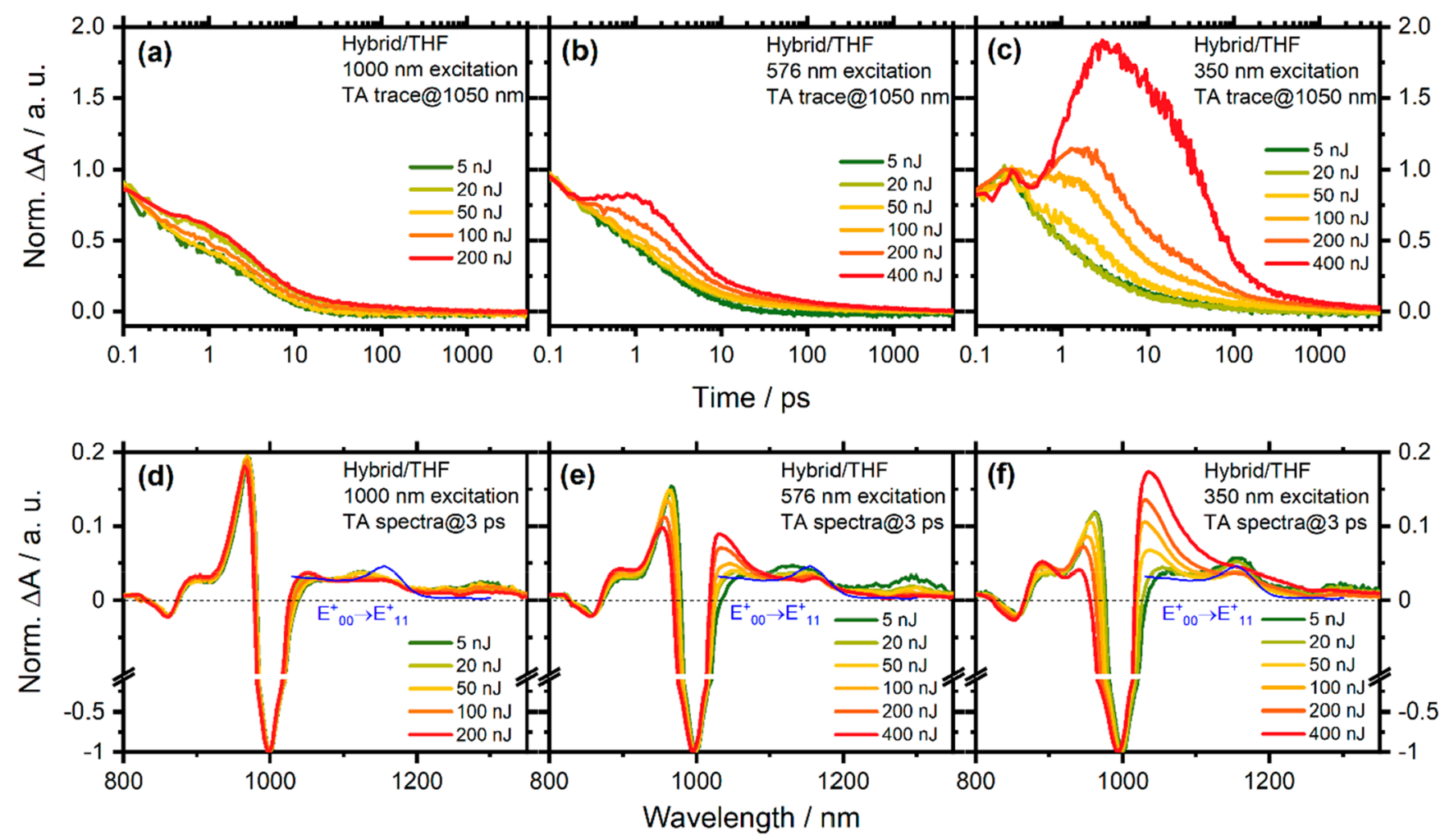

Figure 7. Normalized pump-energy-dependent TA traces at $1050 \mathrm{~nm}$ for the Hybrid in THF upon (a) $\mathrm{E}_{11}$, (b) $\mathrm{E}_{22}$, and (c) $\mathrm{E}_{33}$ excitation. Note that traces were normalized by the $\Delta A$ amplitude at $0.1-0.2$ ps considering the instrumental response. Normalized pump-energy-dependent TA spectra for the Hybrid in THF at a time delay of $\sim 3$ ps upon the (d) $E_{11},(e) E_{22}$, and (f) $E_{33}$ excitations. Note that spectra were normalized at the $\mathrm{E}_{00} \rightarrow \mathrm{E}_{11}$ bleaching maximum. The blue lines represent the stationary absorption feature of the $(6,5)$ SWCNT hole-polaron obtained by redoxchemical doping $\left(\left[\mathrm{NOBF}_{4}\right] \sim 128.4 \mu \mathrm{M}\right.$, shown in Figure 6a).

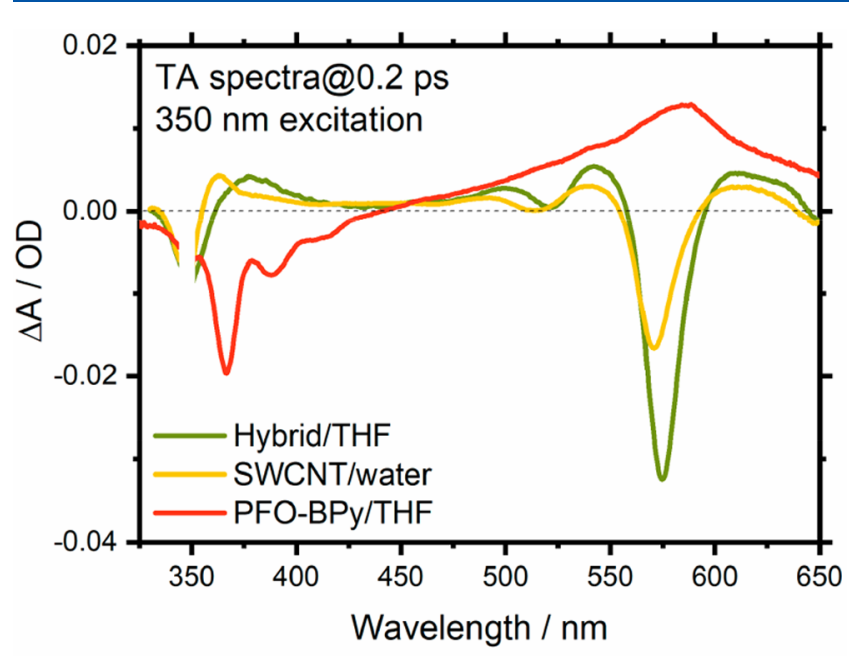

Figure 8. UV-vis TA spectra for the Hybrid in THF, SWCNT in water, and PFO-BPy in THF at a time delay of 0.2 ps. Excitation wavelength: $350 \mathrm{~nm}$. Pump energy: $100 \mathrm{~nJ} \cdot$ pulse $^{-1}$.

excitons, thus making the reaction energetically favorable. For the Hybrid upon $\mathrm{E}_{11}(1000 \mathrm{~nm}, 1.24 \mathrm{eV})$ excitation, however, the annihilation of two $E_{11}$ excitons is energetically insufficient to directly promote an $\mathrm{E}_{33}$ exciton $(350 \mathrm{~nm}, 3.54 \mathrm{eV})$. Consequently, the PCT from the $\mathrm{E}_{11}$-excited $(6,5)$ SWCNT to $\mathrm{PFO}-\mathrm{BPy}$ is quite inefficient as observed in Figure 7. According to previous reports on fluence-dependent dynamics in SWCNTs, ${ }^{67}$ we conclude from the estimated exciton density (see Supporting Information, section I) that the exciton- exciton Auger process is unlikely to occur under $\mathrm{E}_{22}$ excitation with less than $20 \mathrm{~nJ} \cdot$ pulse $^{-1}$. As shown in Figure 7, the PCT characteristics are hardly observable under these conditions.

Due to the effect of exciton dissociation in SWCNTs, the elementary excitation specifically involved in the Auger recombination may not be limited to excitons but can also involve unbound charge carriers, i.e., electrons and holes (e, h). The up-converted product in the Auger recombination of charge carriers is a high-energy carrier (electron or hole), while the product in the Auger recombination of excitons is a highenergy exciton (schematically shown in Figure 9a). It has been shown that these two mechanisms can be distinguished by their distinct population kinetics. ${ }^{34}$ Auger recombination of charge carriers, as a three-particle process, can be described by a rate equation as

$$
\frac{\mathrm{d} n_{\mathrm{e}, \mathrm{h}}(t)}{\mathrm{d} t}=-\frac{1}{3} \gamma_{\mathrm{A}} n_{\mathrm{e}, \mathrm{h}}^{3}(t)
$$

where $n_{\mathrm{e}, \mathrm{h}}(t)$ is the population of charge carriers, and $\gamma_{\mathrm{A}}$ is the rate constant of the Auger recombination of charge carriers. The solution of eq $1,\left[n_{\mathrm{e}, \mathrm{h}}(0) / n_{\mathrm{e}, \mathrm{h}}(t)\right]^{2}-1=2 / 3 \gamma_{\mathrm{A}} n_{\mathrm{e}, \mathrm{h}}^{2}(0) t$, where $n_{e, h}(0)$ denotes the initial population of charge carriers, predicts a linear dependence between the reciprocal of the charge carrier population squared and the delay time. Auger recombination of excitons, as a two-particle process, can be described as

$$
\frac{\mathrm{d} n_{\mathrm{ex}}(t)}{\mathrm{d} t}=-\frac{1}{2} \gamma_{\mathrm{EEA}} n_{\mathrm{ex}}^{2}(t)
$$


(a)

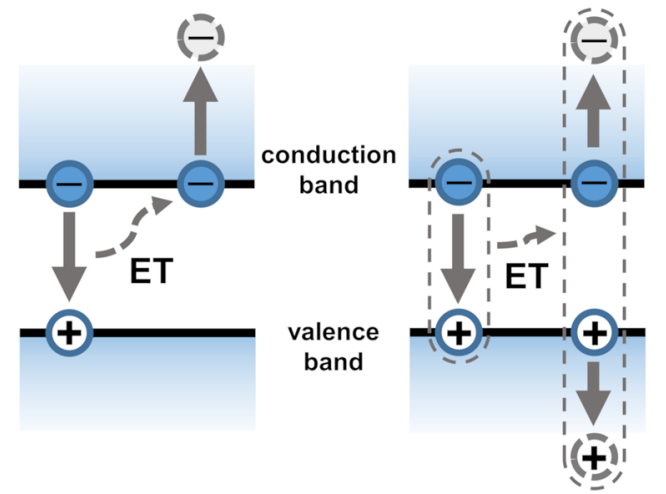

Auger recombination of charge carriers

Auger recombination of excitons

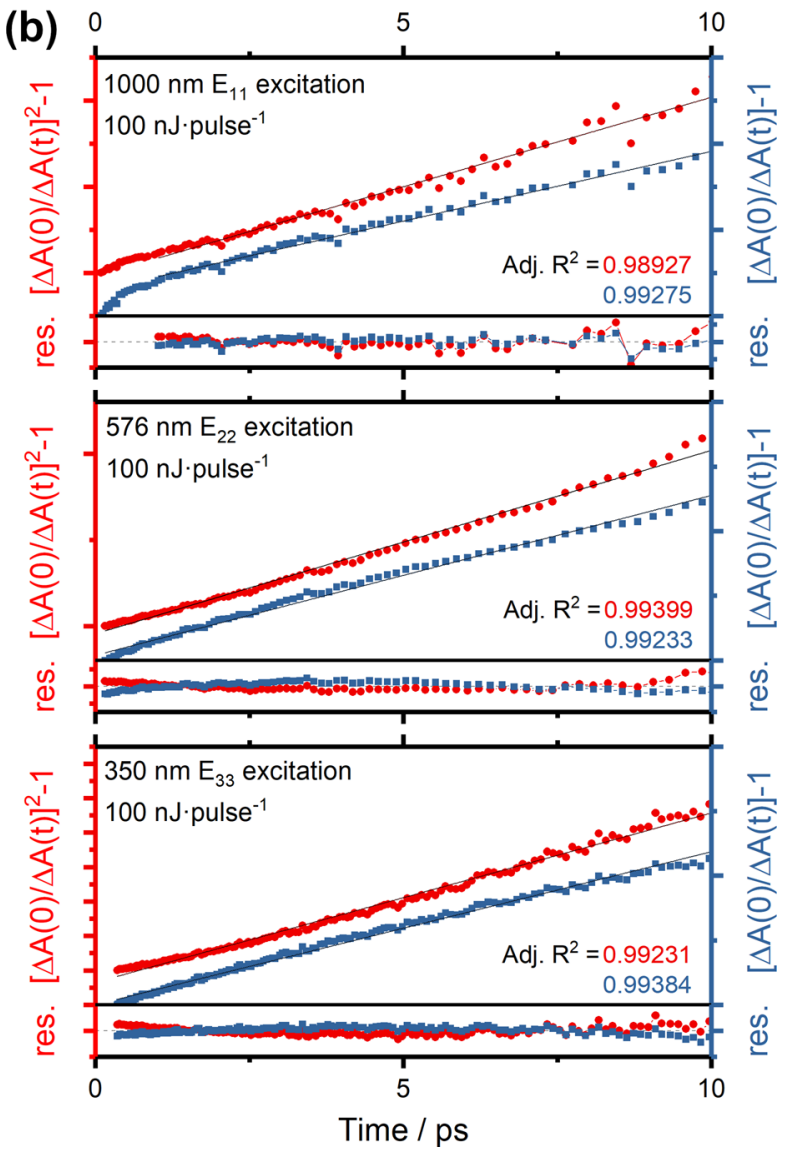

Figure 9. (a) Schematic description of the Auger recombination of charge carriers (left) and excitons (right). ET denotes energy transfer. (b) Kinetics of the integral $\mathrm{E}_{00} \rightarrow \mathrm{E}_{11}$ bleaching in the TA spectra of the Hybrid in THF upon the $\mathrm{E}_{11}, \mathrm{E}_{22}$, and $\mathrm{E}_{33}$ excitations, plotted as $\left\{[\Delta A(0) / \Delta A(t)]^{2}-1\right\}$ (red dots, left axis) and $\{[\Delta A(0) / \Delta A(t)]-$ $1\}$ (blue squares, right axis). Traces are shifted by different offset on the vertical axis for a better comparison. Solid black lines represent the results of the linear fitting. Adjusted $R$-squared (Adj. $R^{2}$ ) and fitting residuals are shown with corresponding colors. Note that the fitting of the $\mathrm{E}_{11}$-excited $\mathrm{TA}$ trace started from 1 ps to exclude the nondiffusion-controlled rapid annihilation. ${ }^{35}$ Pump energy: $100 \mathrm{~nJ}$. pulse ${ }^{-1}$.

where $n_{\mathrm{ex}}(t)$ is the population of excitons, and $\gamma_{\mathrm{EEA}}$ is the rate constant of the Auger recombination of excitons. The solution of eq $2,\left[n_{\mathrm{ex}}(0) / n_{\mathrm{ex}}(t)\right]-1=1 / 2 \gamma_{\mathrm{EEA}} n_{\mathrm{ex}}(0) t$, where $n_{\mathrm{ex}}(0)$ denotes the initial population of excitons, predicts a linear dependence between the reciprocal of the exciton population and the delay time.

To analyze the time dependence of the bleach signal in the initial 10 ps of Hybrid upon $E_{11}, E_{22}$, and $E_{33}$ excitations, the $\mathrm{E}_{00} \rightarrow \mathrm{E}_{11}$ bleaching band in the TA signal is integrated, and its kinetics are plotted as shown in Figure 9b (pump energies of $100 \mathrm{~nJ} \cdot$ pulse $^{-1}$ ) and section G (pump energies of 2-400 nJ. pulse ${ }^{-1}$ ) in the Supporting Information. The linear fitting of $[\Delta A(0) / \Delta A(\mathrm{t})]$ and $[\Delta A(0) / \Delta A(\mathrm{t})]^{2}$ shows, however, comparable adjusted $R$-squared and fitting residuals for all excitation photon energies and excitation fluences. This result suggests that Auger recombination in the Hybrid in the initial $10 \mathrm{ps}$ is not purely excitonic, but the Auger recombination of charge carriers coexists as well.

It is worth noting that when the pump photon energy increases from $1.24 \mathrm{eV}\left(1000 \mathrm{~nm}, \mathrm{E}_{11}\right.$ excitation) to $2.15 \mathrm{eV}$ $\left(576 \mathrm{~nm}, \mathrm{E}_{22}\right.$ excitation $)$ and $3.54 \mathrm{eV}\left(350 \mathrm{~nm}, \mathrm{E}_{33}\right.$ excitation $)$, the charge carrier nature does not obviously replace the excitonic nature as dominant in the elementary excitations of the Hybrid in THF. Besides, the trion, as an indication of efficient free carrier generation (FCG), is absent as well in the TA spectra of the undoped Hybrid as discussed above. All these results indicate that the FCG of the Hybrid in THF is relatively inefficient. The exciton binding energy in carbon nanotubes increases with decreasing solvent dielectric permittivity, ${ }^{68}$ which entails that FCG is highly sensitive to the electrostatic environment. Efficient FCG in pristine SWCNTs is usually observed in high dielectric permittivity microenvironments, such as polar solvents, ionic surfactants, or ionic semiconducting polymers. ${ }^{33,38,69,70}$ Therien et al. pointed out the adverse effect of the low dielectric environment on FCG in SWCNTs by quantitatively analyzing the FCG efficiency in mixtures of $\mathrm{D}_{2} \mathrm{O}(\varepsilon=78.5)$ and $\mathrm{MeOH}(\varepsilon=$ 32.6). ${ }^{38}$ Therefore, we deduce that the FCG in the Hybrid may be suppressed by the low dielectric solvent, THF ( $\varepsilon=$ 7.5). The wrapping PFO-BPy, as a weakly polar polymer, also provides a low dielectric microenvironment for SWCNTs, which is unfavorable to efficient FCG as well. The influence of environmental effects on all-optical FCG in polymer-wrapped SWCNTs still needs further study.

Beside Auger recombination, high-energy and high-fluence excitations may also lead to other nonlinear effects in SWCNTs. The multiple-exciton generation (MEG), as the opposite process of the Auger recombination, forms two excitons by absorbing one photon with energy higher than twice the bandgap. ${ }^{36}$ MEG is, however, an intrinsic feature of SWCNTs and should thus be observable in both surfactantdispersed and polymer-wrapped SWCNTs. The lack of any obvious changes in the line shape of the spectra caused by MEG upon $\mathrm{E}_{33}$ excitation at high pump fluence for pure SWCNTs shows that MEG is not present. Furthermore, MEG as an energy down-conversion process should not contribute to the PCT from excited SWCNTs to wide-bandgap PFO-BPy. Finally, while two-photon absorption (TPA) is an energy upconversion process which may favor the PCT, TPA coefficients in pristine semiconducting SWCNTs have been reported to be very low. ${ }^{71}$

Charge Separation and Recombination Dynamics. Finally, reaction time constants for photoexcited interfacial charge separation $\left(\tau_{\mathrm{CS}}\right)$ and thermal charge recombination $\left(\tau_{\mathrm{CR}}\right)$ in the SWCNT/PFO-BPy Hybrid were estimated by fitting the fluence-dependent kinetic traces (see Supporting Informaton, section $\mathrm{H}$ ). The buildup of the SWCNT polaron 
signature takes place in $\tau_{\mathrm{CS}} \approx 0.9 \mathrm{ps}$, which is consistent with the time scale of reported electron transfer in perylenediimidebased polymer-wrapped carbon nanotube superstructures $\left(\tau_{\mathrm{CS}}\right.$ $\approx 0.4 \mathrm{ps}) .^{22}$ The decay of the SWCNT polaron manifests in a multiphase process, which was reproduced by three-exponential kinetics $\left(\tau_{\mathrm{CR}} \approx 3,50\right.$, and $\left.1000 \mathrm{ps}\right)$. However, the UV-vis TA spectra of the Hybrid corroborate that the decay of the $\mathrm{PFO}-\mathrm{BPy}$ polaron is on a time scale of a few picoseconds and does not feature a nanosecond-lived component (see Supporting Information, section E). The charge recombination path should not be limited to the direct recombination between the SWCNT hole polaron and PFO-BPy electron polaron. The Type-I heterojunction can efficiently funnel the electron on the LUMO of PFO-BPy into the conduction band of the SWCNT, which is independent of whether the SWCNT is charged or neutral. Consequently, the $\mathrm{PFO}-\mathrm{BPy}$ polaron features a significantly shorter decay time compared with that of the SWCNT polaron. When electrons back-transfer to neutral SWCNTs, SWCNT electron polarons will be formed and coexist with the hole polarons because of the high migration rate and large delocalization length of the SWCNT polaron. ${ }^{25,72}$ Therefore, we deduce that the fast decay of the SWCNT polaron on a time scale of $\sim 3$ ps might originate from the direct charge recombination between the closely associated SWCNT electron polaron and PFO-BPy hole polaron. The delocalized SWCNT electron and hole polarons, which migrate along the nanotubes backbone, prolong the final charge recombination to the subnanosecond time scale. ${ }^{22,73-75}$

Through comparison of the spectra of the SWCNT polaron produced by chemical oxidation and the maximal $\mathrm{E}_{00} \rightarrow \mathrm{E}_{11}$ blue-shift in the TA spectra (see Supporting Information, section J), we estimate that PCT induces a doping level higher than the equivalent of $\sim 8 \mu \mathrm{M} \mathrm{NOBF}$ in the SWCNTs. However, because of the spectral overlap in the TA spectra, especially at the delay time when the population of the SWCNT polaron reaches its maximum at around $1-3 \mathrm{ps}$, we could not extract the pure $\mathrm{E}_{00} \rightarrow \mathrm{E}_{11}$ bleach of the SWCNT polaron. Thus, the degree of charge transfer in the PCT reaction cannot be quantified precisely in this work. The question could be addressed in the future through TA spectroscopy on polymer-SWCNTs in thin-film electrochromic devices. ${ }^{6}$

\section{CONCLUSION}

In summary, we have investigated the charge transfer from photoexcited semiconducting $(6,5)$ SWCNTs to a widebandgap wrapping polymer PFO-BPy via femtosecond TA spectroscopy. By spectral and dynamic analysis of the PCT products, we show that the PCT from excited SWCNTs to PFO-BPy can be driven not only by the energetically favorable $\mathrm{E}_{33}$ excitation but also by the energetically unfavorable $\mathrm{E}_{22}$ excitation under high excitation fluences. The energetically unfavorable PCT originates from the Auger recombination of excitons and charge carriers in the SWCNT, which promotes higher energy excitonic states $\left(E_{33}\right.$ or higher) and thus makes the charge transfer from the photoexcited narrow-bandgap SWCNT toward the wide-bandgap polymer possible. The spectral dynamics of the SWCNT polaron indicate a time constant of $\sim 0.9$ ps for the interfacial charge separation reaction between the SWCNT and PFO-BPy. The charge recombination may manifest in multiple paths. It includes the direct recombination between the closely associated SWCNT electron polaron and PFO-BPy hole polaron on a time scale of $\sim 3$ ps, while the delocalized SWCNT electron and hole polarons prolong the final charge recombination to the subnanosecond time scale. These findings expand our understanding of the PCT mechanism in Type-I heterojunctions with SWCNTs. When an energy upconversion process, such as Auger recombination of excitons, takes place in a hybrid system, the energetically unfavorable PCT from a narrow-bandgap nanotube donor to a widebandgap polymer acceptor can be driven as well. Such processes might need to be considered for optoelectronic devices that rely on charge separation between nanotubes and semiconducting polymers (e.g., solar cells or photodiodes), in particular in systems where the polymer bandgap is much smaller than that of PFO-BPy.

\section{ASSOCIATED CONTENT}

\section{Supporting Information}

The Supporting Information is available free of charge at https://pubs.acs.org/doi/10.1021/acs.jpcc.0c10171.

Excitonic transitions in the exciton picture, stationary absorption spectra, pump pulse spectra, fluence-dependent NIR transient absorption spectra of SWCNT and SWCNT/PFO-BPy hybrid, transient absorption spectra of $\mathrm{PFO}-\mathrm{BPy}, \mathrm{PFO}-\mathrm{BPy}$ polaron in $\mathrm{UV}-$ vis transient absorption spectra of the SWCNT/PFO$\mathrm{BPy}$ hybrid and spectroelectrochemistry, elementary excitation analysis of the Auger process, analysis of charge-transfer dynamics, exciton density estimate, and comparison between all-optical doping and chemical doping (PDF)

\section{AUTHOR INFORMATION}

\section{Corresponding Author}

Tiago Buckup - Physikalisch Chemisches Institut and Centre for Advanced Materials, Ruprecht-Karls Universität Heidelberg, Heidelberg 69120, Germany; 이이.org/00000002-1194-0837; Phone: +49 6221 548723;

Email: tiago.buckup@pci.uni-heidelberg.de; Fax: +49 6221548730

\section{Authors}

Zhuoran Kuang - Physikalisch Chemisches Institut and Centre for Advanced Materials, Ruprecht-Karls Universität Heidelberg, Heidelberg 69120, Germany

Felix J. Berger - Physikalisch Chemisches Institut and Centre for Advanced Materials, Ruprecht-Karls Universität Heidelberg, Heidelberg 69120, Germany

Jose Luis Pérez Lustres - Physikalisch Chemisches Institut and Centre for Advanced Materials, Ruprecht-Karls Universität Heidelberg, Heidelberg 69120, Germany

Nikolaus Wollscheid - Physikalisch Chemisches Institut and Centre for Advanced Materials, Ruprecht-Karls Universität Heidelberg, Heidelberg 69120, Germany

Han Li - Institute of Nanotechnology, Karlsruhe Institute of Technology, Eggenstein-Leopoldshafen 76344, Germany

Jan Lüttgens - Physikalisch Chemisches Institut and Centre for Advanced Materials, Ruprecht-Karls Universität Heidelberg, Heidelberg 69120, Germany

Merve Balcl Leinen - Physikalisch Chemisches Institut and Centre for Advanced Materials, Ruprecht-Karls Universität Heidelberg, Heidelberg 69120, Germany 
Benjamin S. Flavel - Institute of Nanotechnology, Karlsruhe Institute of Technology, Eggenstein-Leopoldshafen 76344, Germany; $\odot$ orcid.org/0000-0002-8213-8673

Jana Zaumseil - Physikalisch Chemisches Institut and Centre for Advanced Materials, Ruprecht-Karls Universität Heidelberg, Heidelberg 69120, Germany; ○ orcid.org/00000002-2048-217X

Complete contact information is available at: https://pubs.acs.org/10.1021/acs.jpcc.0c10171

\section{Notes}

The authors declare no competing financial interest.

\section{ACKNOWLEDGMENTS}

This project received funding from the European Research Council under the European Union's Horizon 2020 research and innovation programme (Grant No. 817494). J.L. acknowledges support by the Volkswagenstiftung (Grant No. 93404). M.B.L. received funding from the Deutsche Forschungsgemeinschaft (DFG, Grant No. ZA 638/7). B.S.F. gratefully acknowledges support from the Deutsche Forschungsgemeinschaft (DFG, Grant No. FL 834/2-2, FL 834/5-1, and FL 834/ 7-1).

\section{REFERENCES}

(1) Nish, A.; Hwang, J.-Y.; Doig, J.; Nicholas, R. J. Highly selective dispersion of single-walled carbon nanotubes using aromatic polymers. Nat. Nanotechnol. 2007, 2 (10), 640-646.

(2) Graf, A.; Zakharko, Y.; Schießl, S. P.; Backes, C.; Pfohl, M.; Flavel, B. S.; Zaumseil, J. Large scale, selective dispersion of long single-walled carbon nanotubes with high photoluminescence quantum yield by shear force mixing. Carbon 2016, 105, 593-599.

(3) Samanta, S. K.; Fritsch, M.; Scherf, U.; Gomulya, W.; Bisri, S. Z.; Loi, M. A. Conjugated Polymer-Assisted Dispersion of Single-Wall Carbon Nanotubes: The Power of Polymer Wrapping. Acc. Chem. Res. 2014, 47 (8), 2446-2456.

(4) Rother, M.; Brohmann, M.; Yang, S.; Grimm, S. B.; Schiessl, S. P.; Graf, A.; Zaumseil, J. Aerosol-Jet Printing of Polymer-Sorted (6,5) Carbon Nanotubes for Field-Effect Transistors with High Reproducibility. Adv. Electron. Mater. 2017, 3 (8), 1700080.

(5) Graf, A.; Murawski, C.; Zakharko, Y.; Zaumseil, J.; Gather, M. C. Infrared Organic Light-Emitting Diodes with Carbon Nanotube Emitters. Adv. Mater. 2018, 30 (12), 1706711.

(6) Berger, F. J.; Higgins, T. M.; Rother, M.; Graf, A.; Zakharko, Y.; Allard, S.; Matthiesen, M.; Gotthardt, J. M.; Scherf, U.; Zaumseil, J. From Broadband to Electrochromic Notch Filters with Printed Monochiral Carbon Nanotubes. ACS Appl. Mater. Interfaces 2018, 10 (13), 11135-11142.

(7) Ye, Y.; Bindl, D. J.; Jacobberger, R. M.; Wu, M.-Y.; Roy, S. S.; Arnold, M. S. Semiconducting Carbon Nanotube Aerogel Bulk Heterojunction Solar Cells. Small 2014, 10 (16), 3299-3306.

(8) Pfohl, M.; Glaser, K.; Graf, A.; Mertens, A.; Tune, D. D.; Puerckhauer, T.; Alam, A.; Wei, L.; Chen, Y.; Zaumseil, J.; et al. Probing the Diameter Limit of Single Walled Carbon Nanotubes in SWCNT: Fullerene Solar Cells. Adv. Energy Mater. 2016, 6 (21), 1600890.

(9) Li, G.; Suja, M.; Chen, M.; Bekyarova, E.; Haddon, R. C.; Liu, J.; Itkis, M. E. Visible-Blind UV Photodetector Based on Single-Walled Carbon Nanotube Thin Film/ZnO Vertical Heterostructures. ACS Appl. Mater. Interfaces 2017, 9 (42), 37094-37104.

(10) Schuettfort, T.; Nish, A.; Nicholas, R. J. Observation of a type II heterojunction in a highly ordered polymer-carbon nanotube nanohybrid structure. Nano Lett. 2009, 9 (11), 3871-6.

(11) Eckstein, A.; Karpicz, R.; Augulis, R.; Redeckas, K.; Vengris, M.; Namal, I.; Hertel, T.; Gulbinas, V. Excitation quenching in polyfluorene polymers bound to $(6,5)$ single-wall carbon nanotubes. Chem. Phys. 2016, 467, 1-5.

(12) Amori, A. R; Hou, Z.; Krauss, T. D. Excitons in Single-Walled Carbon Nanotubes and Their Dynamics. Annu. Rev. Phys. Chem. 2018, 69, 81-99.

(13) Bindl, D. J.; Arnold, M. S. Efficient Exciton Relaxation and Charge Generation in Nearly Monochiral $(7,5)$ Carbon Nanotube/ C60 Thin-Film Photovoltaics. J. Phys. Chem. C 2013, 117 (5), 23902395.

(14) Wang, J.; Peurifoy, S. R.; Bender, M. T.; Ng, F.; Choi, K.-S.; Nuckolls, C.; Arnold, M. S. Non-fullerene Acceptors for Harvesting Excitons from Semiconducting Carbon Nanotubes. J. Phys. Chem. C 2019, 123 (35), 21395-21402.

(15) Mollahosseini, M.; Karunaratne, E.; Gibson, G. N.; Gascon, J. A.; Papadimitrakopoulos, F. Fullerene-Assisted Photoinduced Charge Transfer of Single-Walled Carbon Nanotubes through a Flavin Helix. J. Am. Chem. Soc. 2016, 138 (18), 5904-15.

(16) Balcı Leinen, M.; Berger, F. J.; Klein, P.; Mühlinghaus, M.; Zorn, N. F.; Settele, S.; Allard, S.; Scherf, U.; Zaumseil, J. DopingDependent Energy Transfer from Conjugated Polyelectrolytes to $(6,5)$ Single-Walled Carbon Nanotubes. J. Phys. Chem. C 2019, 123 (36), 22680-22689.

(17) Stranks, S. D.; Yong, C.-K.; Alexander-Webber, J. A.; Weisspfennig, C.; Johnston, M. B.; Herz, L. M.; Nicholas, R. J. Nanoengineering Coaxial Carbon Nanotube-Dual-Polymer Heterostructures. ACS Nano 2012, 6 (7), 6058-6066.

(18) Kang, H. S.; Sisto, T. J.; Peurifoy, S.; Arias, D. H.; Zhang, B.; Nuckolls, C.; Blackburn, J. L. Long-Lived Charge Separation at Heterojunctions between Semiconducting Single-Walled Carbon Nanotubes and Perylene Diimide Electron Acceptors. J. Phys. Chem. C 2018, 122 (25), 14150-14161.

(19) Stranks, S. D.; Weisspfennig, C.; Parkinson, P.; Johnston, M. B.; Herz, L. M.; Nicholas, R. J. Ultrafast charge separation at a polymersingle-walled carbon nanotube molecular junction. Nano Lett. 2011, 11 (1), 66-72.

(20) Niklas, J.; Holt, J. M.; Mistry, K.; Rumbles, G.; Blackburn, J. L.; Poluektov, O. G. Charge Separation in P3HT:SWCNT Blends Studied by EPR: Spin Signature of the Photoinduced Charged State in SWCNT. J. Phys. Chem. Lett. 2014, 5 (3), 601-606.

(21) Ferguson, A. J.; Blackburn, J. L.; Holt, J. M.; Kopidakis, N.; Tenent, R. C.; Barnes, T. M.; Heben, M. J.; Rumbles, G. Photoinduced Energy and Charge Transfer in P3HT:SWNT Composites. J. Phys. Chem. Lett. 2010, 1 (15), 2406-2411.

(22) Olivier, J. H.; Park, J.; Deria, P.; Rawson, J.; Bai, Y.; Kumbhar, A. S.; Therien, M. J. Unambiguous Diagnosis of Photoinduced Charge Carrier Signatures in a Stoichiometrically Controlled Semiconducting Polymer-Wrapped Carbon Nanotube Assembly. Angew. Chem., Int. Ed. 2015, 54 (28), 8133-8138.

(23) Kahmann, S.; Salazar Rios, J. M.; Zink, M.; Allard, S.; Scherf, U.; Dos Santos, M. C.; Brabec, C. J.; Loi, M. A. Excited-State Interaction of Semiconducting Single-Walled Carbon Nanotubes with Their Wrapping Polymers. J. Phys. Chem. Lett. 2017, 8 (22), 56665672.

(24) Dabera, G. D.; Jayawardena, K. D.; Prabhath, M. R.; Yahya, I.; Tan, Y. Y.; Nismy, N. A.; Shiozawa, H.; Sauer, M.; Ruiz-Soria, G.; Ayala, P.; et al. Hybrid carbon nanotube networks as efficient hole extraction layers for organic photovoltaics. ACS Nano 2013, 7 (1), $556-65$.

(25) Deria, P.; Olivier, J. H.; Park, J.; Therien, M. J. Potentiometric, electronic, and transient absorptive spectroscopic properties of oxidized single-walled carbon nanotubes helically wrapped by ionic, semiconducting polymers in aqueous and organic media. J. Am. Chem. Soc. 2014, 136 (40), 14193-9.

(26) Wu, J.; Zhang, S.; Lin, D.; Ma, B.; Yang, L.; Zhang, S.; Kang, L.; Mao, N.; Zhang, N.; Tong, L.; et al. Anisotropic Raman-Enhancement Effect on Single-Walled Carbon Nanotube Arrays. Adv. Mater. Interfaces 2018, 5 (3), 1700941. 
(27) Erck, A.; Sapp, W.; Kilina, S.; Kilin, D. Photoinduced Charge Transfer at Interfaces of Carbon Nanotube and Lead Selenide Nanowire. J. Phys. Chem. C 2016, 120 (40), 23197-23206.

(28) Maruyama, S. 1D DOS (van Hove singularity). http://www. photon.t.u-tokyo.ac.jp/ maruyama/kataura/1D_DOS.htmlhttp:// www.photon.t.u-tokyo.ac.jp/ maruyama/kataura/1D_DOS.html (accessed March 26, 2020).

(29) Park, K. H.; Lee, S.-H.; Toshimitsu, F.; Lee, J.; Park, S. H.; Tsuyohiko, F.; Jang, J.-W. Gate-enhanced photocurrent of $(6,5)$ single-walled carbon nanotube based field effect transistor. Carbon 2018, 139, 709-715.

(30) Manzoni, C.; Gambetta, A.; Menna, E.; Meneghetti, M.; Lanzani, G.; Cerullo, G. Intersubband exciton relaxation dynamics in single-walled carbon nanotubes. Phys. Rev. Lett. 2005, 94 (20), 207401.

(31) Crochet, J. J.; Hoseinkhani, S.; Luer, L.; Hertel, T.; Doorn, S. K.; Lanzani, G. Free-carrier generation in aggregates of single-wall carbon nanotubes by photoexcitation in the ultraviolet regime. Phys. Rev. Lett. 2011, 107 (25), 257402.

(32) Park, J.; Reid, O. G.; Blackburn, J. L.; Rumbles, G. Photoinduced spontaneous free-carrier generation in semiconducting single-walled carbon nanotubes. Nat. Commun. 2015, 6, 8809.

(33) Soavi, G.; Scotognella, F.; Brida, D.; Hefner, T.; Späth, F.; Antognazza, M. R.; Hertel, T.; Lanzani, G.; Cerullo, G. Ultrafast Charge Photogeneration in Semiconducting Carbon Nanotubes. J. Phys. Chem. C 2013, 117 (20), 10849-10855.

(34) Valkunas, L.; Ma, Y.-Z.; Fleming, G. R. Exciton-exciton annihilation in single-walled carbon nanotubes. Phys. Rev. B: Condens. Matter Mater. Phys. 2006, 73 (11), 115432.

(35) Ma, Y. Z.; Valkunas, L.; Dexheimer, S. L.; Bachilo, S. M.; Fleming, G. R. Femtosecond spectroscopy of optical excitations in single-walled carbon nanotubes: evidence for exciton-exciton annihilation. Phys. Rev. Lett. 2005, 94 (15), 157402.

(36) Wang, S.; Khafizov, M.; Tu, X.; Zheng, M.; Krauss, T. D. Multiple exciton generation in single-walled carbon nanotubes. Nano Lett. 2010, 10 (7), 2381-6.

(37) O'Connell, M. J.; Bachilo, S. M.; Huffman, C. B.; Moore, V. C.; Strano, M. S.; Haroz, E. H.; Rialon, K. L.; Boul, P. J.; Noon, W. H.; Kittrell, C.; et al. Band Gap Fluorescence from Individual SingleWalled Carbon Nanotubes. Science 2002, 297 (5581), 593-596.

(38) Bai, Y.; Bullard, G.; Olivier, J. H.; Therien, M. J. Quantitative Evaluation of Optical Free Carrier Generation in Semiconducting Single-Walled Carbon Nanotubes. J. Am. Chem. Soc. 2018, 140 (44), 14619-14626.

(39) Park, J.; Deria, P.; Olivier, J. H.; Therien, M. J. Fluencedependent singlet exciton dynamics in length-sorted chirality-enriched single-walled carbon nanotubes. Nano Lett. 2014, 14 (2), 504-11.

(40) Huang, L.; Krauss, T. D. Quantized Bimolecular Auger Recombination of Excitons in Single-Walled Carbon Nanotubes. Phys. Rev. Lett. 2006, 96 (5), 057407.

(41) Li, H.; Gordeev, G.; Garrity, O.; Reich, S.; Flavel, B. S. Separation of Small-Diameter Single-Walled Carbon Nanotubes in One to Three Steps with Aqueous Two-Phase Extraction. ACS Nano 2019, 13 (2), 2567-2578.

(42) Pfohl, M.; Tune, D. D.; Graf, A.; Zaumseil, J.; Krupke, R.; Flavel, B. S. Fitting Single-Walled Carbon Nanotube Optical Spectra. ACS Omega 2017, 2 (3), 1163-1171.

(43) Lüer, L.; Hoseinkhani, S.; Polli, D.; Crochet, J.; Hertel, T.; Lanzani, G. Size and mobility of excitons in $(6,5)$ carbon nanotubes. Nat. Phys. 2009, 5 (1), 54-58.

(44) Styers-Barnett, D. J.; Ellison, S. P.; Mehl, B. P.; Westlake, B. C.; House, R. L.; Park, C.; Wise, K. E.; Papanikolas, J. M. Exciton dynamics and biexciton formation in single-walled carbon nanotubes studied with femtosecond transient absorption spectroscopy. J. Phys. Chem. C 2008, 112 (12), 4507-4516.

(45) Park, J.; Deria, P.; Therien, M. J. Dynamics and transient absorption spectral signatures of the single-wall carbon nanotube electronically excited triplet state. J. Am. Chem. Soc. 2011, 133 (43), 17156-9.
(46) Korovyanko, O. J.; Sheng, C. X.; Vardeny, Z. V.; Dalton, A. B.; Baughman, R. H. Ultrafast spectroscopy of excitons in single-walled carbon nanotubes. Phys. Rev. Lett. 2004, 92 (1), 017403.

(47) Bai, Y.; Olivier, J. H.; Bullard, G.; Liu, C.; Therien, M. J. Dynamics of charged excitons in electronically and morphologically homogeneous single-walled carbon nanotubes. Proc. Natl. Acad. Sci. U. S. A. 2018, 115 (4), 674-679.

(48) Zhu, Z.; Crochet, J.; Arnold, M. S.; Hersam, M. C.; Ulbricht, H.; Resasco, D.; Hertel, T. Pump-Probe Spectroscopy of Exciton Dynamics in $(6,5)$ Carbon Nanotubes. J. Phys. Chem. C 2007, 111 (10), 3831-3835.

(49) Ma, Y.-Z.; Stenger, J.; Zimmermann, J.; Bachilo, S. M.; Smalley, R. E.; Weisman, R. B.; Fleming, G. R. Ultrafast carrier dynamics in single-walled carbon nanotubes probed by femtosecond spectroscopy. J. Chem. Phys. 2004, 120 (7), 3368-3373.

(50) Arias, D. H.; Sulas-Kern, D. B.; Hart, S. M.; Kang, H. S.; Hao, J.; Ihly, R.; Johnson, J. C.; Blackburn, J. L.; Ferguson, A. J. Effect of nanotube coupling on exciton transport in polymer-free monochiral semiconducting carbon nanotube networks. Nanoscale 2019, 11 (44), 21196-21206.

(51) Figueroa Del Valle, D. G.; Moretti, L.; Maqueira-Albo, I.; Aluicio-Sarduy, E.; Kriegel, I.; Lanzani, G.; Scotognella, F. Ultrafast Hole Transfer from $(6,5)$ SWCNT to P3HT:PCBM Blend by Resonant Excitation. J. Phys. Chem. Lett. 2016, 7 (17), 3353-8.

(52) Verissimo-Alves, M.; Capaz, R. B.; Koiller, B.; Artacho, E.; Chacham, H. Polarons in Carbon Nanotubes. Phys. Rev. Lett. 2001, 86 (15), 3372-3375.

(53) Jakubka, F.; Grimm, S. B.; Zakharko, Y.; Gannott, F.; Zaumseil, J. Trion Electroluminescence from Semiconducting Carbon Nanotubes. ACS Nano 2014, 8 (8), 8477-8486.

(54) Matsunaga, R.; Matsuda, K.; Kanemitsu, Y. Observation of charged excitons in hole-doped carbon nanotubes using photoluminescence and absorption spectroscopy. Phys. Rev. Lett. 2011, 106 (3), 037404.

(55) Eckstein, K. H.; Oberndorfer, F.; Achsnich, M. M.; Schöppler, F.; Hertel, T. Quantifying Doping Levels in Carbon Nanotubes by Optical Spectroscopy. J. Phys. Chem. C 2019, 123 (49), 3000130006.

(56) Heller, I.; Kong, J.; Williams, K. A.; Dekker, C.; Lemay, S. G. Electrochemistry at Single-Walled Carbon Nanotubes: The Role of Band Structure and Quantum Capacitance. J. Am. Chem. Soc. 2006, 128 (22), 7353-7359.

(57) Zheng, M.; Diner, B. A. Solution Redox Chemistry of Carbon Nanotubes. J. Am. Chem. Soc. 2004, 126 (47), 15490-15494.

(58) Eckstein, K. H.; Hartleb, H.; Achsnich, M. M.; Schoppler, F.; Hertel, T. Localized Charges Control Exciton Energetics and Energy Dissipation in Doped Carbon Nanotubes. ACS Nano 2017, 11 (10), 10401-10408.

(59) Hartleb, H.; Späth, F.; Hertel, T. Evidence for Strong Electronic Correlations in the Spectra of Gate-Doped Single-Wall Carbon Nanotubes. ACS Nano 2015, 9 (10), 10461-10470.

(60) Kochi, J. K. Inner-sphere electron transfer in organic chemistry. Relevance to electrophilic aromatic nitration. Acc. Chem. Res. 1992, 25 (1), 39-47.

(61) Connelly, N. G.; Geiger, W. E. Chemical Redox Agents for Organometallic Chemistry. Chem. Rev. 1996, 96 (2), 877-910.

(62) Aguirre, C. M.; Levesque, P. L.; Paillet, M.; Lapointe, F.; StAntoine, B. C.; Desjardins, P.; Martel, R. The Role of the Oxygen/ Water Redox Couple in Suppressing Electron Conduction in FieldEffect Transistors. Adv. Mater. 2009, 21 (30), 3087-3091.

(63) Avery, A. D.; Zhou, B. H.; Lee, J.; Lee, E.-S.; Miller, E. M.; Ihly, R.; Wesenberg, D.; Mistry, K. S.; Guillot, S. L.; Zink, B. L.; et al. Tailored semiconducting carbon nanotube networks with enhanced thermoelectric properties. Nat. Energy 2016, 1 (4), 16033.

(64) Holt, J. M.; Ferguson, A. J.; Kopidakis, N.; Larsen, B. A.; Bult, J.; Rumbles, G.; Blackburn, J. L. Prolonging Charge Separation in P3HT-SWNT Composites Using Highly Enriched Semiconducting Nanotubes. Nano Lett. 2010, 10 (11), 4627-4633. 
(65) Sato, K.; Saito, R.; Jiang, J.; Dresselhaus, G.; Dresselhaus, M. S. Discontinuity in the family pattern of single-wall carbon nanotubes. Phys. Rev. B: Condens. Matter Mater. Phys. 2007, 76 (19), 195446.

(66) Chmeliov, J.; Narkeliunas, J.; Graham, M. W.; Fleming, G. R.; Valkunas, L. Exciton-exciton annihilation and relaxation pathways in semiconducting carbon nanotubes. Nanoscale 2016, 8 (3), 16181626.

(67) Harrah, D. M.; Schneck, J. R.; Green, A. A.; Hersam, M. C.; Ziegler, L. D.; Swan, A. K. Intensity-Dependent Exciton Dynamics of $(6,5)$ Single-Walled Carbon Nanotubes: Momentum Selection Rules, Diffusion, and Nonlinear Interactions. ACS Nano 2011, 5 (12), 9898-9906.

(68) Perebeinos, V.; Tersoff, J.; Avouris, P. Scaling of Excitons in Carbon Nanotubes. Phys. Rev. Lett. 2004, 92 (25), 257402.

(69) Santos, S. M.; Yuma, B.; Berciaud, S.; Shaver, J.; Gallart, M.; Gilliot, P.; Cognet, L.; Lounis, B. All-Optical Trion Generation in Single-Walled Carbon Nanotubes. Phys. Rev. Lett. 2011, 107 (18), 187401.

(70) Soavi, G.; Scotognella, F.; Viola, D.; Hefner, T.; Hertel, T.; Cerullo, G.; Lanzani, G. High energetic excitons in carbon nanotubes directly probe charge-carriers. Sci. Rep. 2015, 5, 9681.

(71) Shi, J.; Chu, H.; Li, Y.; Zhang, X.; Pan, H.; Li, D. Synthesis and nonlinear optical properties of semiconducting single-walled carbon nanotubes at $1 \mu \mathrm{m}$. Nanoscale 2019, 11 (15), 7287-7292.

(72) Brady, G. J.; Joo, Y.; Singha Roy, S.; Gopalan, P.; Arnold, M. S. High performance transistors via aligned polyfluorene-sorted carbon nanotubes. Appl. Phys. Lett. 2014, 104 (8), 083107.

(73) Ehli, C.; Oelsner, C.; Guldi, D. M.; Mateo-Alonso, A.; Prato, M.; Schmidt, C.; Backes, C.; Hauke, F.; Hirsch, A. Manipulating single-wall carbon nanotubes by chemical doping and charge transfer with perylene dyes. Nat. Chem. 2009, 1 (3), 243-9.

(74) Dowgiallo, A.-M.; Mistry, K. S.; Johnson, J. C.; Blackburn, J. L. Ultrafast Spectroscopic Signature of Charge Transfer between SingleWalled Carbon Nanotubes and $\mathrm{C}_{60}$. ACS Nano 2014, 8 (8), 85738581.

(75) Howard, I. A.; Mauer, R.; Meister, M.; Laquai, F. Effect of Morphology on Ultrafast Free Carrier Generation in Polythiophene:Fullerene Organic Solar Cells. J. Am. Chem. Soc. 2010, 132 (42), 14866-14876. 